\title{
Generalized Current Green's Function Formalism for Electromagnetic Radiation by Coupled Conducting-Dielectric Systems
}

\author{
Said Mikki \\ Department of Electrical and Computer Engineering \& Computer Science, \\ University of New Haven, West Haven, Connecticut, United States \\ E-mail: said.m.mikki@gmail.com
}

\begin{abstract}
We provide an explicit generalisation of the antenna current Green's function (ACGF) formalism from the perfect electric conducting (PEC) to generic coupled $N$-body systems composed of arbitrarily shaped PEC and dielectric objects, with general permitivity and permeability tensors. Assuming only electromagnetic reciprocity and typical regularity conditions, we construct the current Green's function using the surface equivalence theorem and a combination of other methods involving Riemannian geometry, distribution theory, functional analysis, and the electromagnetic boundary-value problem. The formalism is more complicated than the standard PEC formulation published before due to the need to explicitly account for the interaction between the magnetic field and material interfaces on one hand, and the electric field contribution to conducting parts of the system. For the case of $N$-body system, the ACGF becomes a large tensor array. While its numerical values depend on the coordinate system on the manifolds supporting the electromagnetic boundary conditions, the construction eventually produces coordinate-independent integral expressions for the induced current. Using the ACGF formalism, it is now possible to treat arbitrary $N$-body coupled PEC-dielectric configurations as space-frequency linear systems using exact and rigorous response function, the current Green's function itself. While the derivation is very general, it still leaves open questions regarding whether the ACGF can be constructed for nonreciprocal systems or using volume integral equations.
\end{abstract}

\section{Introduction}

The current Green's function of a material electromagnetic system is defined as the surface current distribution transfer function dictating how an induced current is generated in response to an external electromagnetic field [1]. The concept itself is rooted in earlier circuit representations of antennas as "admittance transfer matrices" (a discrete approximation) $[2,3]$ and is formally identical to the nonlocal response by low-dimensional materials to external electromagnetic fields, for example, lightmatter interactions in nanotubes, graphene, and other novel nano-surfaces [4-6]. In conventional classical electrodynamics, radiation by mico- or macro-sources is often described by the dyadic Green's function of free space where a given radiating current $\mathbf{J}(\mathbf{r})$ is taken as an input while the electromagnetic fields $\mathbf{E}(\mathbf{r})$ and $\mathbf{H}(\mathbf{r})$ produced by this source are the output (the "radiating electromagnetic system.") [7-12]. Following 
a proposal originally stated by Schelkunoff within the framework of transmission line circuit theory [13] but not rigoursly implemented within exact electromagnetic theory till very recently, it was shown that radiation of electromagnetic system is much better described as the cascade of two distinct linear electromagnetic processes, Modes $\mathrm{A}$ and $\mathrm{B}$, where in Mode $\mathrm{A}$ we first induce a current $\mathbf{J}(\mathbf{r})$ by an external field excitation $\mathbf{E}^{\mathrm{ex}}(\mathbf{r})$, e.g., the antenna source or the illumination field in scattering problems, while in Mode B this induced current is allowed to radiate according to the classical dyadic Green's function. The former process, Mode A, is described by a new dyadic Green's function, often denoted by $\overline{\mathbf{F}}\left(\mathbf{r}, \mathbf{r}^{\prime}\right)$ [14-16]. If the traditional radiation dyadic Green's function is given by $\overline{\mathbf{G}}\left(\mathbf{r}, \mathbf{r}^{\prime}\right)$, then the two dyadic Green's functions $\overline{\mathbf{F}}\left(\mathbf{r}, \mathbf{r}^{\prime}\right)$ and $\overline{\mathbf{G}}\left(\mathbf{r}, \mathbf{r}^{\prime}\right)$ together constitute a complete space-frequency representation of the electromagnetic radiation problem describing how a macroscopic material structure responds to an external field by producing another field. This formulation is more natural because it allows a uniform treatment of a wider class electromagnetic problems as input-output systems with the same signal type present at both the excitation and response "terminals": i.e., electromagnetic fields [1]. Applications of the ACGF method in engineering spans topics like mutual coupling analysis [17], mutual coupling compensation [18-20], fast EM computation of large system [21-23], alternative methods to compute the near-field response [24], and wireless communications [25-27] (more applications are discussed in [1].) Most research has been focusing so far on applications with little emphasis on the theoretical, physical, and mathematical foundations, especially for general radiating systems. This paper will be leaning more toward the fundamental theoretical aspects of the generalized ACGF formalism. Readers interested in numerical and experimental results can consult the references quoted above but also see Sec. 3 and the references quoted therein.

From the viewpoint of mathematical physics, the current Green's functions represent a new species of Green's function fundamentally different from other types like those associated with the heat kennel or the wave function, though significant similarities also exist in several other aspects. The most important technical difference is that the current Green's function is the Green's function of the inverse of a global integro-differential operator, while the traditional Green's functions of mathematical physics are those associated with the inversion of a differential - hence local operators. This makes the analysis inherently more complicated and less concrete since no exact analytical expressions in the case of the current Green's function are available and one must resort to a creative - rather nontrivial - combination of analytical and numerical methods. However, the ultimate mathematical foundations of the antenna current Green's function (ACGF) formalism itself were made available by means of a mixture of functional analysis, distribution theory, and Riemannian geometry $[1,15]$. Careful analysis has also revealed recently that because of the global nature of the inverse operator associated with the ACGF, considerations related to causality enters into the picture of spacetime electromagnetic systems [27]. Some additional differences include that the ACGF is essentially a Green's function on a curved Riemannian manifold, while the Green's functions of mathematical physics are typically constructed on flat Euclidean spaces. While an extensive mathematical theory of operators on manifolds and the partial differential equations living on them already exists in the literature, e.g., see $[28,29]$, we note that most of these researches focus on differential operators on manifolds. The case of nonlocal operators globally defined on the entire manifold is more complex since it requires careful analysis of 
the topology of the underlying manifold. Indeed, the global geometry of the radiating surface enters into the behaviour of the response function at every point. It has been already suggested in $[15,16]$ that this deep connection between the topology and geometry of the radiating surface and the electromagnetic response is one of the most fundamental applications of the ACGF formalism to the physics of electromagnetic systems.

Even though the ACGF formalism has been utilized in the analysis and design of various antenna systems $[1,14-16,20,21,24,27,30]$, including mixed PEC-dielectric configurations $[18,22,23]$, to the best of our knowledge there currently exists no published account of how the formalism can be derived and formulated for the general case of multiple PEC and dielectric objects existing simultaneously in the system. Moreover, even the single-dielectric type case, i.e., one all-dielectric inhomogeneous domain in PEC-free system, has never been treated in print anywhere. This is in direct contrast to the extreme importance of mixed PEC-dielectric radiating (and receiving) systems, most prominently of course printed antenna systems such as patches and planner dipoles, and volumetric-type dielectric resonator antennas. In addition, highpower radiators like horn antennas are sometimes loaded with dielectric media in order to control their radiation characteristics, making them another example of antenna types that need to be handled using an expanded or generalized ACGF formalism going beyond the now standard PEC-based ACGF theory. This paper focuses exclusively on the purely mathematical aspects of the generalized ACGF formalism, in addition to extended discussion of the physical and conceptual aspects related to the mathematical relations derived here. No detailed discussion of the computational and experimental applications will be given here since these have been treated at length elsewhere. However, we provide toward the end of this paper (Sec. 3) a brief discussion of the literature on the computational implementation of the ACGF method and how it is related to some applications. Readers more interested in applications may consult Part 4 in the book [1], in addition to some of the more recent literature, such as [19,25,27].

Our mathematical treatment will be schematic and the rigid formal theorem-proof format will not be followed here to increase the accessibility of the work to wider audience.

\section{Exact Derivation of the Generalized ACGF Tensor Array in Generic PEC-Dielectric Systems}

\subsection{Background Preparation: The Operator Formalism of Minimal Coupled PEC-Dielectric Systems}

Throughout this paper, we assume a time harmonic excitation of the form $\exp (i \omega t)$. In other words, we develop here a frequency-domain (space-frequency) current Green's function formalism, while the generalization to spacetime is left for future work. Let us first consider the minimal system composed of two material types, one PEC, and the other electric-magnetic type described by electric permitivity function $\overline{\bar{\varepsilon}}(\omega)$ and permeability $\overline{\bar{\mu}}(\omega)$, where both are allowed to be functions of the operating frequency $\omega . \ddagger$ The system is depicted in Fig. 1, where the externally applied fields $\mathbf{E}^{\mathrm{ex}}(\mathbf{r})$ and $\mathbf{H}^{\mathrm{ex}}(\mathbf{r})$, while $\mathbf{E}^{s}(\mathbf{r})$ and $\mathbf{H}^{s}(\mathbf{r})$ are the secondary (scattered) fields. The total fields

$\ddagger$ Here, with the risk of abusing the common terminology, we include magnetic properties in those material domains we called 'dielectric'. 
are given by

$$
\mathbf{E}(\mathbf{r})=\mathbf{E}^{\mathrm{ex}}(\mathbf{r})+\mathbf{E}^{s}(\mathbf{r}), \mathbf{H}(\mathbf{r})=\mathbf{H}^{\mathrm{ex}}(\mathbf{r})+\mathbf{H}^{s}(\mathbf{r}) .
$$

For simplicity, throughout this paper each dielectric domain is assumed to be homogeneous, so we drop out any possible dependence of the material profiles on position within each sub-region. $\S$ The region supporting the dielectric medium is denoted by $V_{d}$ with enclosing surface $S_{d}$, while the PEC region has the corresponding $V_{c}$ and $S_{c}$, respectively. All enclosing surfaces are assumed to enjoy enough regularity to allow the use of standard vector calculus and function analysis, e.g., see [31,32]. Associated with each closed 2-manifold $S_{d}$ and $S_{c}$ is a smooth normal unit vector field $\hat{u}(\mathbf{r})$, with $\mathbf{r}$ in either $S_{c}$ of $S_{d}$, which is always clear from the context. All regions will be embedded into an infinite free space exterior domain $V_{\text {ext }}:=\mathbb{R}^{3}-V_{d}-V_{c}$, with permitivity and permeability $\varepsilon_{0}$ and $\mu_{0}$. We will assume the applicability of the radiation condition at infinitely, and hence the surface $S_{\infty}$ is not mentioned here. In what follows, the relevant integral representation theorems [33] will be used in conjunction with this radiation condition since we are interested in working with scattering/radiation problems.
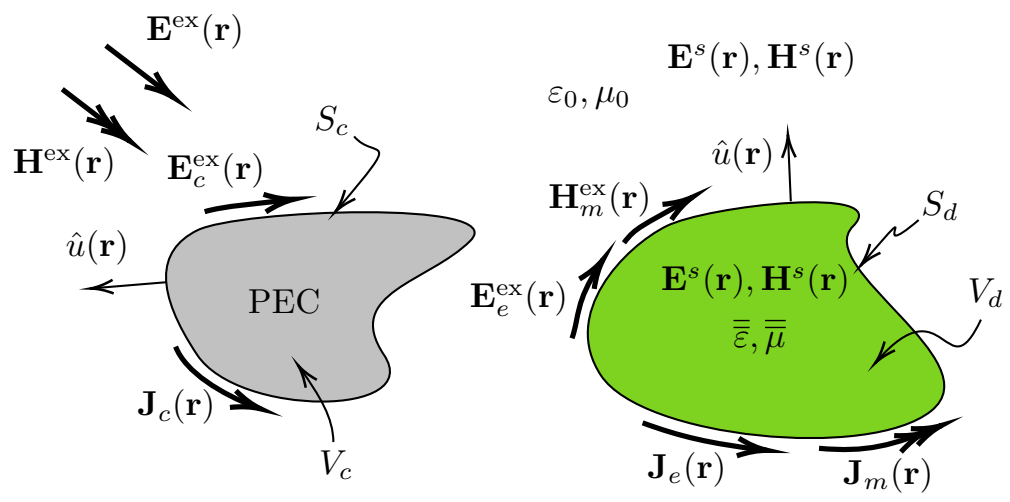

Figure 1: Minimal coupled PEC-dielectric system composed of exactly one PEC subdomain $V_{c}$ enclosed by surface $S_{c}=\partial V_{c}$, and exactly one dielectric subdomain $V_{d}$ with boundary $S_{d}=\partial V_{d}$. The dielectric subdomain electric and magnetic properties are described by permitivity and permeability tensors $\overline{\bar{\varepsilon}}(\omega), \overline{\bar{\mu}}(\omega)$, respectively, and these are assumed to be reciprocal. The surfaces $S_{c}$ and $S_{d}$ are modeled as oreintable differential manifold with the Riemannian metric inherited from the embedding ambient Euclidean space $\mathbb{R}^{3}$. We note that the total fields

The electromagnetic boundary conditions are $[10,34]$

$$
\hat{u}(\mathbf{r}) \times \mathbf{E}(\mathbf{r})=0, \quad \mathbf{r} \in S_{c},
$$

for the PEC domain, and the following continuity relations of the tangential electric and magnetic fields, respectively, for the dielectric domains:

$$
\hat{u}(\mathbf{r}) \times \mathbf{E}\left(\mathbf{r}^{+}\right)=\hat{u}(\mathbf{r}) \times \mathbf{E}\left(\mathbf{r}^{-}\right), \hat{u}(\mathbf{r}) \times \mathbf{H}\left(\mathbf{r}^{+}\right)=\hat{u}(\mathbf{r}) \times \mathbf{H}\left(\mathbf{r}^{-}\right), \mathbf{r} \in S_{d}-S_{c} .
$$

$\S$ That does not constitute a serious loss of generality since we will later generalize the formalism to deal with arbitrary number of sub-domains. Since inhomogeneous regions can be approximated (at least in first attempts) by a finite number of smaller humongous microdomains, the generic $N$-domain formalism of this paper should be sufficient for a large number of practical applications. 
From these boundary conditions and using the standard surface equivalence theorem $[10,35]$, we introduce three types of currents in this system: conducting currents $\mathbf{J}_{c}(\mathbf{r})$, electric currents $\mathbf{J}_{e}(\mathbf{r})$, and magnetic currents $\mathbf{J}_{m}(\mathbf{r})$ given by [35-37]

$$
\begin{gathered}
\mathbf{J}_{c}(\mathbf{r}):= \begin{cases}\hat{u}(\mathbf{r}) \times \mathbf{H}(\mathbf{r}), & \mathbf{r} \in S_{c}, \\
0, & \text { otherwise. }\end{cases} \\
\mathbf{J}_{e}(\mathbf{r}):=\left\{\begin{array}{ll}
\hat{u}(\mathbf{r}) \times \mathbf{H}(\mathbf{r}), & \mathbf{r} \in S_{d}, \\
0, & \text { otherwise. }
\end{array}, \quad \mathbf{J}_{m}(\mathbf{r}):=\left\{\begin{array}{cc}
-\hat{u}(\mathbf{r}) \times \mathbf{E}(\mathbf{r}), & \mathbf{r} \in S_{d}, \\
0, & \text { otherwise. }
\end{array} .\right.\right.
\end{gathered}
$$

The first type is supported only at the PEC surface $S_{c}$ and vanishes everywhere else, while the remaining two are supported on the surfaces $S_{d}$. Each of these currents will radiate both electric and magnetic field components into the interior and exterior domains of the problem. In other words, we are using the special form of Schelkunoff's surface equivalence theorem [37] implied in (4) and (5), which gives correct fields only in the exterior region (our main domain of interest in this paper, which focuses on radiation problems), while the internal fields are zero (Love's Principle [36].) However, for this to happen, the entire space of the problem, the unbounded domain $\mathbb{R}^{3}$ must be treated as free space $\varepsilon_{0}, \mu_{0}$ (or the same as the properties of the exterior domain in the original problem if the radiation is not in free space.) This is the exterior domain formulation depicted in Fig. 2 where the presence of the primary external (excitation) fields is included in the exterior domain (but they can also be present in the interior domain with obvious modifications.) On the other hand, the surface equivalent currents $-\mathbf{J}_{e}(\mathbf{r})$ and $-\mathbf{J}_{m}(\mathbf{r})$ will radiate null fields in the exterior domain but produce the correct fields in the interior region $V_{d}$ provided the entire unbounded domain is filled with the medium having $\overline{\bar{\varepsilon}}, \overline{\bar{\mu}}$ as depicted in Fig. 3. In this formulation, the exterior domain fields $\mathbf{E}^{+}(\mathbf{r}), \mathbf{H}^{+}(\mathbf{r})$ in Fig. 2 are identical to the correct original fields $\mathbf{E}(\mathbf{r}), \mathbf{H}(\mathbf{r})$, the latter due to the system Fig. 1 and defined by (1), but only for $\mathbf{r} \in \mathbb{R}^{3}-V_{d}$, while the interior domain fields $\mathbf{E}^{-}(\mathbf{r}), \mathbf{H}^{-}(\mathbf{r})$ in Fig. 3 are equal to the original fields (1) for $\mathbf{r} \in V_{d}$. Finally, using the principle of linear superposition, the total exterior-domain electric and magnetic fields, denoted by $\mathbf{E}(\mathbf{r})$ and $\mathbf{H}(\mathbf{r})$, respectively, are given by

$$
\mathbf{E}(\mathbf{r})=\mathbf{E}^{\mathrm{ex}}(\mathbf{r})+\underbrace{\mathbf{E}_{e}(\mathbf{r})+\mathbf{E}_{m}(\mathbf{r})+\mathbf{E}_{c}(\mathbf{r})}_{\mathbf{E}^{s}(\mathbf{r})}, \mathbf{H}(\mathbf{r})=\mathbf{H}^{\mathrm{ex}}(\mathbf{r})+\underbrace{\mathbf{H}_{e}(\mathbf{r})+\mathbf{H}_{m}(\mathbf{r})+\mathbf{H}_{c}(\mathbf{r})}_{\mathbf{H}^{s}(\mathbf{r})},
$$

Here, $\mathbf{E}^{\mathrm{ex}}(\mathbf{r})$ and $\mathbf{H}^{\mathrm{ex}}(\mathbf{r})$ are the same as the externally supplied excitation in Fig. 1, which are assumed to exist in the exterior region only. In the remaining field symbols, each subscript indicates which source current type contributed to the production of the field component under consideration. For example, $\mathbf{E}_{m}(\mathbf{r})$ is the electric field produced by the magnetic current $\mathbf{J}_{m}(\mathbf{r}), \mathbf{H}_{c}(\mathbf{r})$ is the magnetic field produced by the conducting current $\mathbf{J}_{c}(\mathbf{r})$ etc. Using this notation, the total exterior and interior fields may be expressed as

$$
\begin{aligned}
\mathbf{E}^{+}=\mathbf{E}^{\mathrm{ex}}+\mathbf{E}_{e}^{+}+\mathbf{E}_{m}^{+}+\mathbf{E}_{c}^{+}, & \mathbf{H}^{+}=\mathbf{H}^{\mathrm{ex}}+\mathbf{H}_{e}^{+}+\mathbf{H}_{m}^{+}+\mathbf{H}_{c}^{+}, \\
\mathbf{E}^{-}=\mathbf{E}_{e}^{-}+\mathbf{E}_{m}^{-}, & \mathbf{H}^{-}=\mathbf{H}_{e}^{-}+\mathbf{H}_{m}^{-} .
\end{aligned}
$$

where the convention that $\mathbf{E}^{\mathrm{ex}}(\mathbf{r})=\mathbf{H}^{\mathrm{ex}}(\mathbf{r})=0$ for $\mathbf{r} \in V_{d}$ was used here. Also note that $\mathbf{E}_{c}=\mathbf{H}_{c}=0$ since $\mathbf{J}_{c}=0$ for the interior problem Fig. 3 . 


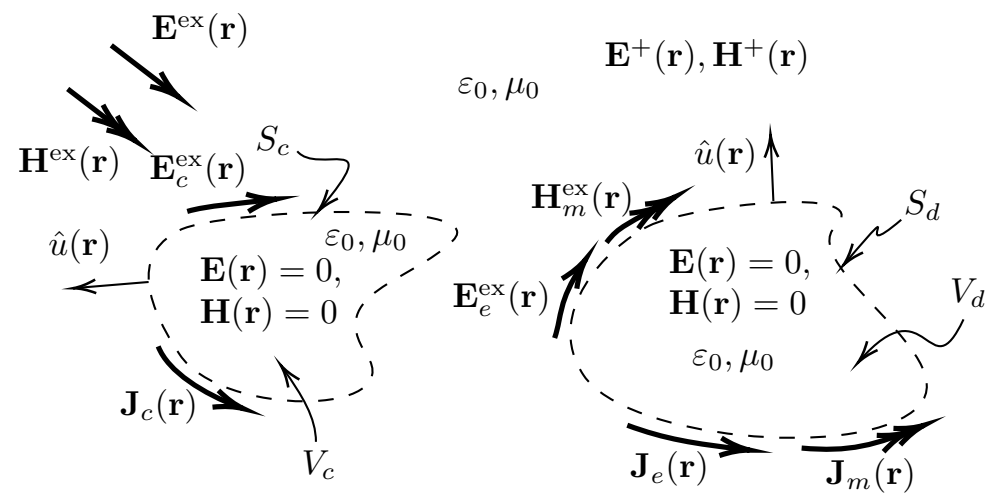

Figure 2: Exterior (radiation) problem reformulation using the surface equivalence theorem (Love-Schelkunoff [37] Principle).

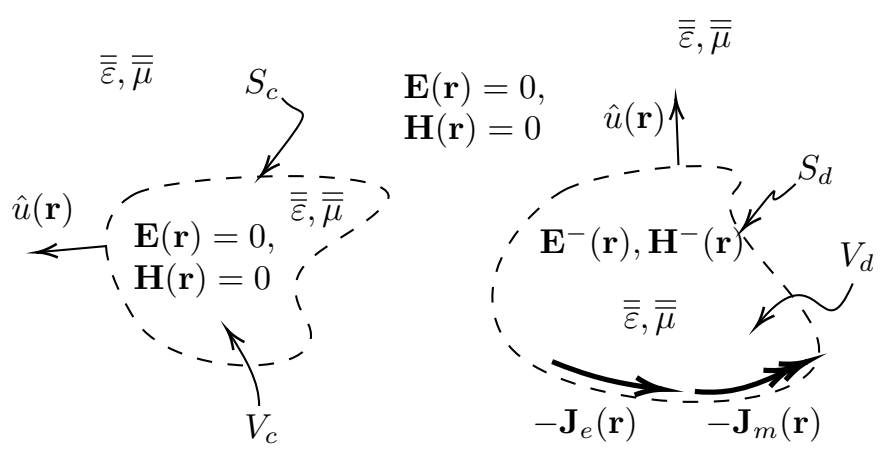

Figure 3: Interior (radiation) problem reformulation using the surface equivalence theorem (Love-Schelkunoff [37] Principle).

Under such conditions of the surface equivalence theorem, the field integral representation theorems can be used to express the total interior and exterior electric and magnetic via the operator relations $[9,10,32-35,38,39]$

$$
\begin{aligned}
& \mathbf{E}_{e}^{ \pm}(\mathbf{r})=\mathcal{G}_{e e}^{ \pm}\left(\mathbf{r}, \mathbf{r}^{\prime}\right) \mathbf{J}_{e}\left(\mathbf{r}^{\prime}\right), \mathbf{H}_{m}^{ \pm}(\mathbf{r})=\mathcal{G}_{m m}^{ \pm}\left(\mathbf{r}, \mathbf{r}^{\prime}\right) \mathbf{J}_{m}\left(\mathbf{r}^{\prime}\right), \\
& \mathbf{E}_{c}^{ \pm}(\mathbf{r})=\mathcal{G}_{e c}^{ \pm}\left(\mathbf{r}, \mathbf{r}^{\prime}\right) \mathbf{J}_{c}\left(\mathbf{r}^{\prime}\right), \mathbf{H}_{e}^{ \pm}(\mathbf{r})=\mathcal{G}_{m e}^{ \pm}\left(\mathbf{r}, \mathbf{r}^{\prime}\right) \mathbf{J}_{e}\left(\mathbf{r}^{\prime}\right), \\
& \mathbf{E}_{m}^{ \pm}(\mathbf{r})=\mathcal{G}_{e m}^{ \pm}\left(\mathbf{r}, \mathbf{r}^{\prime}\right) \mathbf{J}_{m}\left(\mathbf{r}^{\prime}\right), \mathbf{H}_{c}^{ \pm}(\mathbf{r})=\mathcal{G}_{m c}^{ \pm}\left(\mathbf{r}, \mathbf{r}^{\prime}\right) \mathbf{J}_{c}\left(\mathbf{r}^{\prime}\right),
\end{aligned}
$$

Here, the operator $\mathcal{G}_{a b}^{ \pm}\left(\mathbf{r}, \mathbf{r}^{\prime}\right), a \in\{e, m\}, b \in\{e, m, c\}$, is defined by the integral relation (Green's function operator)

$$
\mathcal{G}_{a b}^{ \pm}\left(\mathbf{r}, \mathbf{r}^{\prime}\right) \mathbf{J}_{b}\left(\mathbf{r}^{\prime}\right):=\int_{S(b)} \mathrm{d} s^{\prime} \overline{\mathbf{G}}_{a b}^{ \pm}\left(\mathbf{r}, \mathbf{r}^{\prime}\right) \cdot \mathbf{J}_{b}\left(\mathbf{r}^{\prime}\right),
$$

where

$$
S(b):= \begin{cases}S_{c}, & b=c, \\ S_{d}, & b=e, m .\end{cases}
$$

The quantities $\overline{\mathbf{G}}_{a b}^{+}\left(\mathbf{r}, \mathbf{r}^{\prime}\right)$ and $\overline{\mathbf{G}}_{a b}^{-}\left(\mathbf{r}, \mathbf{r}^{\prime}\right)$ are the dyadic Green's functions for the infinite and unbounded domains filled with materials having $\left(\varepsilon_{0}, \mu_{0}\right),(\overline{\bar{\varepsilon}}, \overline{\bar{\mu}})$, respectively. They 
connect a current excitation of the type $\mathbf{J}_{b}$ with either the electric field when Not all of them are independent; e.g., it is obvious that $\overline{\mathbf{G}}_{a c}^{ \pm}\left(\mathbf{r}, \mathbf{r}^{\prime}\right)=\overline{\mathbf{G}}_{a e}^{ \pm}\left(\mathbf{r}, \mathbf{r}^{\prime}\right)$ for all $a=e, m, c$. Moreover, other dyadic Green's functions are strongly related to each due to the symmetry (duality) relations. Since we will not need the specific forms of these functions in what follows, such additional relations will not be listed here since they are readily available in the literature. $\|$ It is significant to note that the field produced by each current source is obtained via nonlocal relation, i.e., Green's functions operators (8), which are essentially integro-differential operators that can be defined on some convenient Banach or Sobolev space, see [32,33]. We denoted a generic such operator by the symbol $\mathcal{G}_{a b}^{ \pm}$, where $a \in\{e, m\}, b \in\{e, m, c\}$, which can be physically interpreted in the following manner: An entity expressed by $\mathcal{G}_{e m}^{+}$, for example, denotes the radiation operator responsible of the production of the exterior electric field component $\mathbf{E}_{e}$ due to the magnetic current $\mathbf{J}_{m}$ when radiating into an infinite domain filled with $\varepsilon_{0}, \mu_{0}$, etc. Most advanced classical electromagnetic theory books present derivations of these nonlocal operators, which we will not give here, but see $[9,10,32,33,35,38-41]$ for extensive further details and discussion regarding the mathematical theory and the computational aspects of these radiation operators. For the case of generic anisotropic media, the Green's function are less well understood and their computation is considerably more complicated. In general, no close-form expressions exist for $\overline{\mathbf{G}}_{a b}^{-}\left(\mathbf{r}, \mathbf{r}^{\prime}\right)$ in the anisotropic medium scenario, in contrast to the case with $\overline{\mathbf{G}}_{a b}^{+}\left(\mathbf{r}, \mathbf{r}^{\prime}\right)$ where the latter are very well understood since they possess a relatively simple analytical form. It is also worth mentioning that in the engineering community, the dyadic Green's functions of inhomogeneous media, mainly stratified domains, have received very extensive attention and are often formulated and computed efficiently, e.g., see $[9,12,38]$.

Let us now define the surface excitation fields, i.e., the pertinent illumination fields interacting with the system, which originate from an external source. These are constructed in terms of which surface they interact with. As will become apparent immediately, in the generalized ACGF formalism, it is absolutely essential that fields interacting with different boundary types are distinguished from each other. Mostly conspicuous is the case of electric fields, where whether they interact with PEC surface or material interface makes a fundamental difference in the mathematical formulation. The detailed definitions are

$$
\mathbf{E}_{e}^{\mathrm{ex}}(\mathbf{r}):=\mathbf{E}^{\mathrm{ex}}(\mathbf{r}) \psi\left(\mathbf{r} ; S_{d}\right), \mathbf{H}_{m}^{\mathrm{ex}}(\mathbf{r}):=\mathbf{H}^{\mathrm{ex}}(\mathbf{r}) \psi\left(\mathbf{r} ; S_{d}\right), \mathbf{E}_{c}^{\mathrm{ex}}(\mathbf{r}):=\mathbf{E}^{\mathrm{ex}}(\mathbf{r}) \psi\left(\mathbf{r} ; S_{c}\right),
$$

where the surface membership function $\psi: S \rightarrow \mathbb{R}$, denoted by $\psi(\mathbf{r} ; S)$, is given by

$$
\psi(\mathbf{r} ; S):= \begin{cases}1, & \mathbf{r} \in S \\ 0, & \text { otherwise. }\end{cases}
$$

Remember that the magnetic field does not contribute to the ACGF for PEC objects [1]. The use of the membership function (11) is usually replaced in differential topology and mathematical analysis by the process of restriction of the function domain [42]. We may now put together all these notations and general theorems and combine them with the boundry conditions (2) and (3) in conjunction with the two surface

$\|$ We also further note that since in the interior problem of Fig. $3, \mathbf{J}_{c}=0$, the operators $\mathcal{G}_{a c}^{-}$will not be actually used in what follows. 
equivlance problems dpecited in Figs. 2 and 3. Using (8) in the electromagnetic boundary conditions (2) and (3), we find

$$
\begin{gathered}
\hat{u}(\mathbf{r}) \times\left[\mathbf{E}^{\mathrm{ex}}(\mathbf{r})+\mathbf{E}_{e}^{+}(\mathbf{r})+\mathbf{E}_{m}^{+}(\mathbf{r})+\mathbf{E}_{c}^{+}(\mathbf{r})\right]=0, \quad \mathbf{r} \in S_{c} \\
\hat{u}(\mathbf{r}) \times\left[\mathbf{E}^{\mathrm{ex}}(\mathbf{r})+\mathbf{E}_{e}^{+}(\mathbf{r})+\mathbf{E}_{m}^{+}(\mathbf{r})+\mathbf{E}_{c}^{+}(\mathbf{r})\right]_{+}=\hat{u}(\mathbf{r}) \times\left[\mathbf{E}_{e}^{-}(\mathbf{r})+\mathbf{E}_{m}^{-}(\mathbf{r})\right]_{-}, \mathbf{r} \in S_{d} \\
\hat{u}(\mathbf{r}) \times\left[\mathbf{H}^{\mathrm{ex}}(\mathbf{r})+\mathbf{H}_{e}^{+}(\mathbf{r})+\mathbf{H}_{m}^{+}(\mathbf{r})+\mathbf{H}_{c}^{+}(\mathbf{r})\right]_{+}=\hat{u}(\mathbf{r}) \times\left[\mathbf{E}_{e}^{-}(\mathbf{r})+\mathbf{H}_{m}^{-}(\mathbf{r})\right]_{-}, \mathbf{r} \in S_{d} .
\end{gathered}
$$

Here, the subscripts ' + ' and '-' indicate attached to the brackets in the second and third equations indicate that we will be approaching the surface $S_{d}$ from the outside and inside, respectively. In general, jump conditions, which depend on the Green's functions $\overline{\mathbf{G}}_{a b}^{ \pm}\left(\mathbf{r}, \mathbf{r}^{\prime}\right)$ being used, must be employed to further simplify the system of equation (12). Moreover, several combination of electric and magnetic type boundary conditions might be used, leading to electric field integral equations (EFIE), magnetic field integral equation (MFIE), and combined electric-magnetic field integral equation (CIE) [38]. All such cases can be put in the following extremely general system of operator equations $[10,35,38,40,43-45]$

$$
\begin{array}{lc}
\mathcal{L}_{e e}\left(\mathbf{r}, \mathbf{r}^{\prime}\right) \mathbf{J}_{e}\left(\mathbf{r}^{\prime}\right)+\mathcal{L}_{e m}\left(\mathbf{r}, \mathbf{r}^{\prime}\right) \mathbf{J}_{m}\left(\mathbf{r}^{\prime}\right)+\mathcal{L}_{e c}\left(\mathbf{r}, \mathbf{r}^{\prime}\right) \mathbf{J}_{c}\left(\mathbf{r}^{\prime}\right)=\hat{u}\left(\mathbf{r}^{\prime}\right) \times \mathbf{E}_{e}^{\mathrm{ex}}\left(\mathbf{r}^{\prime}\right), & \mathbf{r} \in S_{d} \\
\mathcal{L}_{m e}\left(\mathbf{r}, \mathbf{r}^{\prime}\right) \mathbf{J}_{e}\left(\mathbf{r}^{\prime}\right)+\mathcal{L}_{m m}\left(\mathbf{r}, \mathbf{r}^{\prime}\right) \mathbf{J}_{m}\left(\mathbf{r}^{\prime}\right)+\mathcal{L}_{m c}\left(\mathbf{r}, \mathbf{r}^{\prime}\right) \mathbf{J}_{c}\left(\mathbf{r}^{\prime}\right)=\hat{u}\left(\mathbf{r}^{\prime}\right) \times \mathbf{H}_{m}^{\mathrm{ex}}\left(\mathbf{r}^{\prime}\right), & \mathbf{r} \in S_{d} \\
\mathcal{L}_{c e}\left(\mathbf{r}, \mathbf{r}^{\prime}\right) \mathbf{J}_{e}\left(\mathbf{r}^{\prime}\right)+\mathcal{L}_{c m}\left(\mathbf{r}, \mathbf{r}^{\prime}\right) \mathbf{J}_{m}\left(\mathbf{r}^{\prime}\right)+\mathcal{L}_{c c}\left(\mathbf{r}, \mathbf{r}^{\prime}\right) \mathbf{J}_{c}\left(\mathbf{r}^{\prime}\right)=\hat{u}\left(\mathbf{r}^{\prime}\right) \times \mathbf{E}_{c}^{\mathrm{ex}}\left(\mathbf{r}^{\prime}\right), & \mathbf{r} \in S_{c}
\end{array}
$$

In this system, the unknowns are the three current distributions $\mathbf{J}_{e}(\mathbf{r}), \mathbf{J}_{m}(\mathbf{r})$, and $\mathbf{J}_{c}(\mathbf{r})$, while the input excitation (forcing term) are the fields $\mathbf{E}_{e}^{\mathrm{ex}}\left(\mathbf{r}^{\prime}\right), \mathbf{H}_{m}^{\mathrm{ex}}\left(\mathbf{r}^{\prime}\right)$, and $\mathbf{E}_{c}^{\text {ex }}\left(\mathbf{r}^{\prime}\right)$ defined by (10). The exact form of the operators $\mathcal{L}_{a b}, a, b \in\{e, m, c\}$, depends on the jump conditions and which surface integral equation was used. For the generalized ACGF formalism, as long as the system is solvable the exact details of these sub-operators are not needed, hence the powerful generality of the method. Note that by the way we defined the unit normal vector field $\hat{u}(\mathbf{r})$, which has a support only on the manifolds $S_{c}$ and $S_{d}$, the RHS of (13) is ensured to be nonzero only on either a PEC surface or a material interface. In other words, from the perspective of linear system theory, the equations (13) express the antenna/scattering problem as a linear response problem where input excitation fields, $\mathbf{E}^{\mathrm{ex}}$ and $\mathbf{H}^{\mathrm{ex}}$ interact only with 2-manifolds, here $S_{c}$ and $S_{d}$, in spite of the fact that the interacting fields are 3dimensional spacetime processes. This dimensional change is one of the main reasons why the current Green's function formalism requires extreme care when formulating its main equations and results as was originally noted in [15].

The three linear operator equations in (13) must be solved for the unknown currents simultaneously, very much like in ordinary linear algebraic equations $[38,40]$. This amounts to inverting the global operator of the system, denoted by $\mathcal{L}\left(\mathbf{r}, \mathbf{r}^{\prime}\right)$, which is defined by the following operator relation

$$
\mathcal{L}\left(\mathbf{r}, \mathbf{r}^{\prime}\right) \cdot\left[\mathbf{J}\left(\mathbf{r}^{\prime}\right)\right]=\left[\mathbf{V}^{\mathrm{ex}}(\mathbf{r})\right],
$$

where

$$
[\mathbf{J}(\mathbf{r})]_{9 \times 1}:=\left(\begin{array}{c}
\mathbf{J}_{e}(\mathbf{r}) \\
\mathbf{J}_{m}(\mathbf{r}) \\
\mathbf{J}_{c}(\mathbf{r})
\end{array}\right)
$$


is a compact form of the three unknown vector current distributions, while

$$
\left[\mathbf{V}^{\mathrm{ex}}(\mathbf{r})\right]_{9 \times 1}:=\hat{u}(\mathbf{r}) \times\left(\begin{array}{c}
\mathbf{E}_{e}^{\mathrm{ex}}(\mathbf{r}) \\
\mathbf{H}_{m}^{\mathrm{ex}}(\mathbf{r}) \\
\mathbf{E}_{c}^{\mathrm{ex}}(\mathbf{r})
\end{array}\right)
$$

is the corresponding form of the excitation fields. In component form, the global integro-differential operator of the PEC-dielectric system can be expressed as

$$
\begin{aligned}
& \mathcal{L}\left(\mathbf{r}, \mathbf{r}^{\prime} ; \omega, S, \overline{\bar{\varepsilon}}, \overline{\bar{\mu}}\right):= \\
& \left(\begin{array}{lll}
\mathcal{L}_{e e}\left(\mathbf{r}, \mathbf{r}^{\prime} ; \omega, S, \overline{\bar{\varepsilon}}, \overline{\bar{\mu}}\right) & \mathcal{L}_{e m}\left(\mathbf{r}, \mathbf{r}^{\prime} ; \omega, S, \overline{\bar{\varepsilon}}, \overline{\bar{\mu}}\right) & \mathcal{L}_{e c}\left(\mathbf{r}, \mathbf{r}^{\prime} ; \omega, S, \overline{\bar{\varepsilon}}, \overline{\bar{\mu}}\right) \\
\mathcal{L}_{m e}\left(\mathbf{r}, \mathbf{r}^{\prime} ; \omega, S, \overline{\bar{\varepsilon}}, \overline{\bar{\mu}}\right) & \mathcal{L}_{m m}\left(\mathbf{r}, \mathbf{r}^{\prime} ; \omega, S, \overline{\bar{\varepsilon}}, \overline{\bar{\mu}}\right) & \mathcal{L}_{m c}\left(\mathbf{r}, \mathbf{r}^{\prime} ; \omega, S, \overline{\bar{\varepsilon}}, \overline{\bar{\mu}}\right) \\
\mathcal{L}_{c e}\left(\mathbf{r}, \mathbf{r}^{\prime} ; \omega, S, \overline{\bar{\varepsilon}}, \overline{\bar{\mu}}\right) & \mathcal{L}_{c m}\left(\mathbf{r}, \mathbf{r}^{\prime} ; \omega, S, \overline{\bar{\varepsilon}}, \overline{\bar{\mu}}\right) & \mathcal{L}_{c c}\left(\mathbf{r}, \mathbf{r}^{\prime} ; \omega, S, \overline{\bar{\varepsilon}}, \overline{\bar{\mu}}\right)
\end{array}\right),
\end{aligned}
$$

where we emphasize here the frequency dependence (which will be drop out most of the time in this paper.) The sub-operators appearing in (17) are all functions of the material composition tensors $\overline{\bar{\varepsilon}}$ and $\overline{\bar{\mu}}$ as well as the geometry of the interface manifolds $S$, but mention of this dependence will be also dropped out to simplify the notation though implied.

\subsection{Exact Derivation of the Generalized ACGF Tensor Array in Minimal PEC-Dielectric Systems}

We are ready now to construct the current Green's function of the mixed PECdielectric system using the techniques proposed in [15]. It will be seen that similar to the PEC case, the ACGF of the PEC-dielectric configuration is not an ordinary function, but a distribution, i.e., a generalized function [42, 46, 47]. A Green's function is a response function representing how a linear system reacts to influences or external inputs [42]. However, not every linear system can automatically possess a Green's function, but in each case the Green's function must be explicitly shown to exist $[42,48]$. In mathematical physics, since most physical processes of interest are described by differential equations (partial or ordinary), an extensive literature on the subject of Green's functions for linear partial differential equations has been developed, especially for the most important cases of first and second order systems [49]. In our case, the linear operator system (13) is quite different since the operators appearing in (8) composing the global operator (17) are integro-differential operators, hence not strictly speaking local. This implies that the existence of the current Green's function in this case is not obvious and requires explicit construction. To do so, we follow the same path proposed earlier in [1] where a specialized surface delta function is first defined then later deployed to prove the existence of the ACGF by actually constructing one.

Generalized functions like the Green's function or the Dirac delta function are defined with respect (or found to be equivalent) to equivalence classes of sequences of ordinary functions satisfying certain properties [42,46,47,49-51]. A surface delta function $\delta_{S}\left(\mathbf{r}, \mathbf{r}^{\prime}\right)$ is a delta function living only on the smooth manifold $S$. It is defined as the generalized function [15]

$$
\delta_{S}\left(\mathbf{r}, \mathbf{r}^{\prime}\right): \stackrel{\text { g.f }}{=}\left(d_{1}\left(\mathbf{r}, \mathbf{r}^{\prime} ; S\right) \quad d_{2}\left(\mathbf{r}, \mathbf{r}^{\prime} ; S\right) \quad \cdots \quad d_{n}\left(\mathbf{r}, \mathbf{r}^{\prime} ; S\right) \quad \cdots\right),
$$


where the equality is not point-like as in ordinary function, but to be understood in the sense of equivalence class-type of equality $[46,47,50]$. The functions $d_{n}$, however, are all ordinary smooth functions by design. Explicit expressions for the function sequence $d_{n}\left(\mathbf{r}, \mathbf{r}^{\prime}\right), n=1,2, \cdots$, were given in $[1,15]$, where the Riemannian metric tensor field $g(\mathbf{r})$, describing the inner product on two tangent vectors at $\mathbf{r}$, enters into the definition of $\delta_{S}$. Moreover, due to the inhomogeneous nature of the space described by the smooth manifold $S$, the delta function is not shift-invariant and hence cannot be written as function of $\mathbf{r}-\mathbf{r}^{\prime}$ as in the case with the more familiar Dirac delta functions on Euclidean spaces $\mathbb{R}^{n}$ for positive integers $n$ [47]. It can be shown that $[1,15]$

$$
V(\mathbf{r})=\lim _{n \rightarrow \infty} \int_{S} \mathrm{~d} s^{\prime} d_{n}\left(\mathbf{r}, \mathbf{r}^{\prime} ; S\right) V\left(\mathbf{r}^{\prime}\right)
$$

is valid for any smooth scalar field $V(\mathbf{r})$ on $S$. In fact, one may consider (19) the defining relation of the surface delta function $\delta_{S}$ of which the form (18) is merely its rigorous rendering via distribution theory. Indeed, both (18) and (19) are combined into

$$
V(\mathbf{r})=\int_{S} \mathrm{~d} s^{\prime} \delta_{S}\left(\mathbf{r}, \mathbf{r}^{\prime}\right) V\left(\mathbf{r}^{\prime}\right),
$$

which is the formal relation usually invoked in textbooks as the definition of the Dirac delta function (the sifting property.)

Next, we need to "vectorize" the surface delta function in order to fit the specific needs of the mixed PEC-dielectric ACGF construction under consideration. To do so, let us introduce the vector array

$$
\left[\delta_{\mathrm{S}}^{\mathrm{ex}}(\mathbf{r})\right]:=\left(\begin{array}{c}
\hat{\alpha}_{e} \delta_{S_{d}}\left(\mathbf{r}, \mathbf{r}^{\prime}\right) \\
\hat{\alpha}_{m} \delta_{S_{d}}\left(\mathbf{r}, \mathbf{r}^{\prime}\right) \\
\hat{\alpha}_{c} \delta_{S_{c}}\left(\mathbf{r}, \mathbf{r}^{\prime}\right)
\end{array}\right) .
$$

It captures in a compact form the three independent excitation fields pertinent to the system's current Green's function. Here, the three vectors $\hat{\alpha}_{e}, \hat{\alpha}_{m}$, and $\hat{\alpha}_{c}$ are independent unit vectors encoding the source polarization data, while the impulsive nature of these excitation inputs is represented by the surface delta defined on the proper 2-manifold corresponding to each source. Using (18), the delta source array (21) can be explicated as

$$
\left[\delta_{\mathrm{S}}^{\mathrm{ex}}(\mathbf{r})\right]: \stackrel{: \text {.f }}{=}\left(\left[\begin{array}{llllll}
{\left[\mathbf{f}_{1}^{\mathrm{ex}}(\mathbf{r} ; S)\right]} & {\left[\mathbf{f}_{2}^{\mathrm{ex}}(\mathbf{r} ; S)\right]} & \cdots & {\left[\mathbf{f}_{n}^{\mathrm{ex}}(\mathbf{r} ; S)\right]} & \cdots
\end{array}\right),\right.
$$

where the regular source (ordinary) fields are

$$
\left[\mathbf{f}_{n}^{\mathrm{ex}}(\mathbf{r} ; S)\right]:=\left(\begin{array}{c}
\hat{\alpha}_{e} d_{n}\left(\mathbf{r}, \mathbf{r}^{\prime} ; S_{d}\right) \\
\hat{\alpha}_{m} d_{n}\left(\mathbf{r}, \mathbf{r}^{\prime} ; S_{d}\right) \\
\hat{\alpha}_{c} d_{n}\left(\mathbf{r}, \mathbf{r}^{\prime} ; S_{c}\right)
\end{array}\right)
$$

The surface vector field function (23) represent a manageable ordinary excitation function on which one may apply the normal rules of calculus and functional analysis, in particular the traditional boundary-value problem and its functional analytic formulation as will be seen shortly. 
From classical electromagnetic theory, it can be shown that the general operator relation (14) is invertible under very reasonable restrictions. The details can be found in $[32,33,38]$. Indeed, the mixed PEC-dielectric system under illumination by smooth fields, with regularity conditions imposed on the geometry - already ensured by assuming that $S$ possesses a differential (smooth) manifold structure - is a wellposed boundary-value problem with the proper existence and uniqueness theorems applicable to it. Under these circumstances, we write

$$
[\mathbf{J}(\mathbf{r})]=\mathcal{L}^{-1}\left(\mathbf{r}, \mathbf{r}^{\prime}\right) \cdot\left[\mathbf{V}\left(\mathbf{r}^{\prime}\right)\right],
$$

where $\mathcal{L}^{-1}$ is the inverse $\mathcal{L}^{-1}:=(\mathcal{L})^{-1}$ of the global operator (17) given by

$$
\mathcal{L}^{-1}\left(\mathbf{r}, \mathbf{r}^{\prime}\right)=\left(\begin{array}{ccc}
\mathcal{L}_{e e}^{-1}\left(\mathbf{r}, \mathbf{r}^{\prime}\right) & \mathcal{L}_{e m}^{-1}\left(\mathbf{r}, \mathbf{r}^{\prime}\right) & \mathcal{L}_{e c}^{-1}\left(\mathbf{r}, \mathbf{r}^{\prime}\right) \\
\mathcal{L}_{m e}^{-1}\left(\mathbf{r}, \mathbf{r}^{\prime}\right) & \mathcal{L}_{m m}^{-1}\left(\mathbf{r}, \mathbf{r}^{\prime}\right) & \mathcal{L}_{m c}^{-1}\left(\mathbf{r}, \mathbf{r}^{\prime}\right) \\
\mathcal{L}_{c e}^{-1}\left(\mathbf{r}, \mathbf{r}^{\prime}\right) & \mathcal{L}_{c m}^{-1}\left(\mathbf{r}, \mathbf{r}^{\prime}\right) & \mathcal{L}_{c c}^{-1}\left(\mathbf{r}, \mathbf{r}^{\prime}\right)
\end{array}\right)
$$

Therefore, like the forward global operator, the inverse operator must be an array of nine sub-operators, here denoted by $\mathcal{L}_{a b}^{-1}\left(\mathbf{r}, \mathbf{r}^{\prime}\right), a, b \in\{e, m, c\}$. It should be immediately noted that

$$
\mathcal{L}_{a b}^{-1}\left(\mathbf{r}, \mathbf{r}^{\prime}\right) \neq\left(\mathcal{L}_{a b}\left(\mathbf{r}, \mathbf{r}^{\prime}\right)\right)^{-1}, \quad \forall a, b \in\{e, m, c\} .
$$

In other words, it is not possible to obtain the elements of the individual sub-operators composing $\mathcal{L}^{-1}$ by simply inverting the corresponding original forward operators $\mathcal{L}_{a b}\left(\mathbf{r}, \mathbf{r}^{\prime}\right)$ appearing in (17). This observation is important because it can be easily overlooked. The confusion may arise from the fact that the various forward suboperators $\mathcal{L}_{a b}\left(\mathbf{r}, \mathbf{r}^{\prime}\right)$ possess expressions independent (formally) from each other, and hence one may indeed mathematically invert the individual operators. However, since the three equations (13) must be satisfied simultaneously to ensure the correctness of the proper electromagnetic boundary conditions of the system under consideration, it is necessary to invert the entire operator array (17).

Following $[1,14,15]$, we define the current Green's function (ACGF) of the PECdielectric system by the relation

$$
\left[\overline{\mathbf{F}}\left(\mathbf{r}, \mathbf{r}^{\prime}\right)\right]:=\mathcal{L}^{-1}\left(\mathbf{r}, \mathbf{r}^{\prime}\right) \cdot\left[\delta_{\mathrm{S}}^{\mathrm{ex}}\left(\mathbf{r}^{\prime}\right)\right] .
$$

It should be clear by now that this definition must be ultimately understood in the sense of generalized functions. Hence, by invoking the distributional definition of the excitation field (22), we introduce

$$
\left[\overline{\mathbf{F}}_{n}\left(\mathbf{r}, \mathbf{r}^{\prime}\right)\right]:=\mathcal{L}^{-1}\left(\mathbf{r}, \mathbf{r}^{\prime}\right) \cdot\left[\mathbf{f}_{n}^{\mathrm{ex}}\left(\mathbf{r}^{\prime}\right)\right] .
$$

which is defined as the $n$th level distributional approximation of the ACGF. As can be readily verified, the elements of the function sequence $\left[\overline{\mathbf{F}}_{n}\left(\mathbf{r}, \mathbf{r}^{\prime}\right)\right], n=1,2, \cdots$, are

(i) ordinary smooth functions, and

(ii) tensorial fields defined on the 2-dimensional Riemannian manifold $S$.

Fact (i) implies that all rules of normal vector analysis applies when we work with the distributional approximation functions $\left[\overline{\mathbf{F}}_{n}\left(\mathbf{r}, \mathbf{r}^{\prime}\right)\right], n=1,2, \cdots, \infty$. In particular, this implies that ordinary full-wave numerical methods in computational electromagenetics 
(CEM) can be deployed to calculate these level functions themselves. This observation was heavily exploited in [1] in order to approximate the ACGF of various PEC systems. Moreover, fact (ii) says that the approximating functions $\left[\overline{\mathbf{F}}_{n}\left(\mathbf{r}, \mathbf{r}^{\prime}\right)\right]$, and hence the ACGF itself, have acquired an additional structure, that of a tensor on 2-manifold. Therefore, $\left[\overline{\mathbf{F}}\left(\mathbf{r}, \mathbf{r}^{\prime}\right)\right]$ represents a Green's tensor living in a curved Riemannian manifold $S$. We will come again to the explicit form of this Green's tensor shortly. Putting in all these information together, we may now construct a formal distributional definition of the ACGF through

$$
\left[\overline{\mathbf{F}}\left(\mathbf{r}, \mathbf{r}^{\prime}\right)\right]: \stackrel{\text { g.f }}{=}\left(\left[\begin{array}{lll}
\left.\overline{\mathbf{F}}_{1}\left(\mathbf{r}, \mathbf{r}^{\prime}\right)\right] & {\left[\overline{\mathbf{F}}_{2}\left(\mathbf{r}, \mathbf{r}^{\prime}\right)\right] \quad\left[\overline{\mathbf{F}}_{n}\left(\mathbf{r}, \mathbf{r}^{\prime}\right)\right]} & \cdots
\end{array}\right) .\right.
$$

This is the fundamental distributional constructive definition of the generalized ACGF. Everything to follow is based on it.

With the help of (25), the definition (27) implies that the generalized ACGF should be expanded into an array of nine sub-ACGFs, i.e., $\left[\overline{\mathbf{F}}\left(\mathbf{r}, \mathbf{r}^{\prime}\right)\right]$ possesses the form

$$
\left[\overline{\mathbf{F}}\left(\mathbf{r}, \mathbf{r}^{\prime}\right)\right]=\left(\begin{array}{ccc}
\overline{\mathbf{F}}_{e e}\left(\mathbf{r}, \mathbf{r}^{\prime}\right) & \overline{\mathbf{F}}_{e m}\left(\mathbf{r}, \mathbf{r}^{\prime}\right) & \overline{\mathbf{F}}_{e c}\left(\mathbf{r}, \mathbf{r}^{\prime}\right) \\
\overline{\mathbf{F}}_{m e}\left(\mathbf{r}, \mathbf{r}^{\prime}\right) & \overline{\mathbf{F}}_{m m}\left(\mathbf{r}, \mathbf{r}^{\prime}\right) & \overline{\mathbf{F}}_{m c}\left(\mathbf{r}, \mathbf{r}^{\prime}\right) \\
\overline{\mathbf{F}}_{c e}\left(\mathbf{r}, \mathbf{r}^{\prime}\right) & \overline{\mathbf{F}}_{c m}\left(\mathbf{r}, \mathbf{r}^{\prime}\right) & \overline{\mathbf{F}}_{c c}\left(\mathbf{r}, \mathbf{r}^{\prime}\right)
\end{array}\right) .
$$

In details, the individual ACGFs composing this generalized ACGF array are given by the following expression

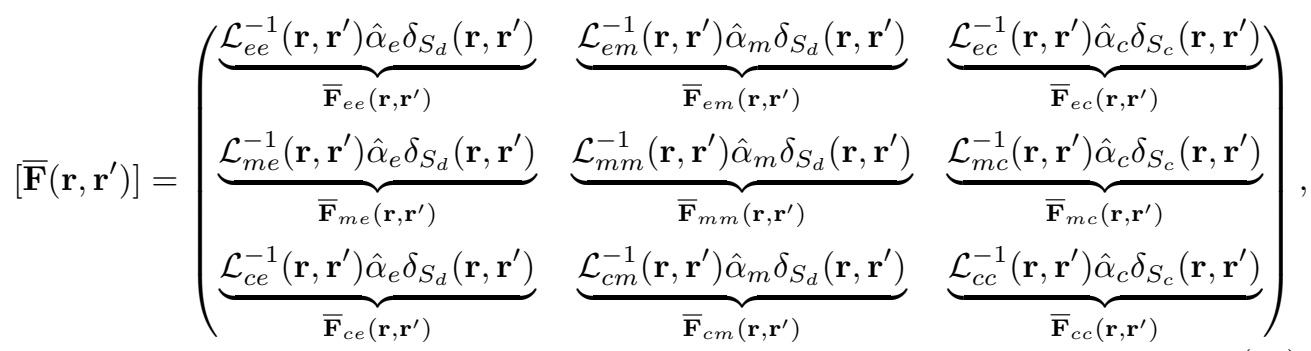

Each ACGF is in itself a tensor on its associated 2-dimensional Riemannian manifold $S$. In order to effectively describe the structure of this tensor. i.e., in a coordinateindependent manner, we need to work with the differential atlas associated with the manifold $S$ on which the ACGF tensor is defined. Let $\left(U_{i}, \varphi_{i}\right), i \in I$, be such an atlas, where $\varphi_{i}: U_{i} \rightarrow \mathbb{R}^{2}$ is the $i$ th coordinate system associated with the open set $U_{i} \subset S$, while $I$ is the index set. The coordinates themselves are given by $x^{n}=\varphi_{i}^{n}(\mathbf{r})$, with $n=1,2$ to account for the two degrees of freedom on a 2-dimensional manifold. The manifold itself is the union $S=\cup_{i \in I} U_{i}$ with possible overlap between individual $U_{i}$. The tangent vector at each point $\mathbf{r} \in U_{i} \subset S$ can be expressed using the coordinates of that patch using the relation $[49,52]$

$$
\hat{\alpha}(\mathbf{r})=\alpha_{1}(\mathbf{r}) \frac{\partial}{\partial x^{1}}+\alpha_{2}(\mathbf{r}) \frac{\partial}{\partial x^{2}} .
$$

This is the expression of the tangent vector $\alpha$ at $\mathbf{r}$ in terms of local (intrinsic) coordinate systems. But we can also expand the same vector in terms of external global Cartesian coordinate system associated with the ambient space $\mathbb{R}^{3}$ into which $S$ is embedded, so we write $\hat{\alpha}_{1}$ and $\hat{\alpha}_{2}$ as the Cartesian form for $\alpha_{1}$ and $\alpha_{2}$, respectively. 
The two vectors are clearly linearly independent but they are not generally required to be orthogonal. For convenience, in this paper we assume that they are further orthonormal, i.e., we have

$$
\hat{\alpha}_{n}(\mathbf{r}) \cdot \hat{\alpha}_{m}(\mathbf{r})=\delta_{n m},
$$

where $\delta_{n m}$ is the Kronecker delta function. Based on this geometric formulation, the ACGF can be readily expanded into dyadic basis in the form $\hat{\alpha}_{i}(\mathbf{r}) \otimes \hat{\alpha}_{j}\left(\mathbf{r}^{\prime}\right)$. If we drop the tensor product sign $\otimes$ for simplicity, we obtain

$$
\begin{aligned}
\overline{\mathbf{F}}_{a b}\left(\mathbf{r}, \mathbf{r}^{\prime}\right) & =\sum_{i, j=1}^{2} \hat{\alpha}_{i}(\mathbf{r}) \hat{\alpha}_{j}\left(\mathbf{r}^{\prime}\right) F_{a b}^{i j}\left(\mathbf{r}, \mathbf{r}^{\prime}\right) \\
& =\left(\begin{array}{ll}
\hat{\alpha}_{1}(\mathbf{r}) \hat{\alpha}_{1}\left(\mathbf{r}^{\prime}\right) F_{a b}^{11}\left(\mathbf{r}, \mathbf{r}^{\prime}\right) & \hat{\alpha}_{1}(\mathbf{r}) \hat{\alpha}_{2}\left(\mathbf{r}^{\prime}\right) F_{a b}^{12}\left(\mathbf{r}, \mathbf{r}^{\prime}\right) \\
\hat{\alpha}_{2}(\mathbf{r}) \hat{\alpha}_{1}\left(\mathbf{r}^{\prime}\right) F_{a b}^{21}\left(\mathbf{r}, \mathbf{r}^{\prime}\right) & \hat{\alpha}_{2}(\mathbf{r}) \hat{\alpha}_{2}\left(\mathbf{r}^{\prime}\right) F_{a b}^{22}\left(\mathbf{r}, \mathbf{r}^{\prime}\right)
\end{array}\right)
\end{aligned}
$$

where $a, b \in\{e, m, c\}$. In Appendix B, all of the concrete generalized ACGF array component expressions are listed in order to spell out which manifolds $\left(S_{d}\right.$ or $\left.S_{c}\right)$ are relevant to each ACGF.

The final - and most crucial - step in the derivation of the generalized ACGF formalism involve demonstrating an analog to the sifting property (20) valid for the current distribution array response $[\mathbf{J}(\mathbf{r})]$. Here, as in [15], it turns out that the proof of the main relation must use reciprocity (consequent on the self-adjointness of the relevant operators). Let us start by considering the $n$-level distributional approximation of the surface current

$$
\left[\mathbf{J}_{n}(\mathbf{r})\right]=\int_{S} \mathrm{~d} s^{\prime}\left[\overline{\mathbf{F}}_{n}\left(\mathbf{r}, \mathbf{r}^{\prime}\right)\right] \cdot\left[\mathbf{V}\left(\mathbf{r}^{\prime}\right)\right] .
$$

Here, the $n$-level ACGF approximation $\left[\overline{\mathbf{F}}_{n}\left(\mathbf{r}, \mathbf{r}^{\prime}\right)\right]$ can be computed with the help of

$$
\begin{aligned}
& {\left[\overline{\mathbf{F}}_{n}\left(\mathbf{r}, \mathbf{r}^{\prime}\right)\right]} \\
& =\left(\begin{array}{lll}
\mathcal{L}_{e e}^{-1}\left(\mathbf{r}, \mathbf{r}^{\prime}\right) \hat{\alpha}_{e} d_{n}\left(\mathbf{r}, \mathbf{r}^{\prime} ; S_{d}\right) & \mathcal{L}_{e m}^{-1}\left(\mathbf{r}, \mathbf{r}^{\prime}\right) \hat{\alpha}_{m} d_{n}\left(\mathbf{r}, \mathbf{r}^{\prime} ; S_{d}\right) & \mathcal{L}_{e c}^{-1}\left(\mathbf{r}, \mathbf{r}^{\prime}\right) \hat{\alpha}_{c} d_{n}\left(\mathbf{r}, \mathbf{r}^{\prime} ; S_{c}\right) \\
\mathcal{L}_{m e}^{-1}\left(\mathbf{r}, \mathbf{r}^{\prime}\right) \hat{\alpha}_{e} d_{n}\left(\mathbf{r}, \mathbf{r}^{\prime} ; S_{d}\right) & \mathcal{L}_{m m}^{-1}\left(\mathbf{r}, \mathbf{r}^{\prime}\right) \hat{\alpha}_{m} d_{n}\left(\mathbf{r}, \mathbf{r}^{\prime} ; S_{d}\right) & \mathcal{L}_{m c}^{-1}\left(\mathbf{r}, \mathbf{r}^{\prime}\right) \hat{\alpha}_{c} d_{n}\left(\mathbf{r}, \mathbf{r}^{\prime} ; S_{c}\right) \\
\mathcal{L}_{c e}^{-1}\left(\mathbf{r}, \mathbf{r}^{\prime}\right) \hat{\alpha}_{e} d_{n}\left(\mathbf{r}, \mathbf{r}^{\prime} ; S_{d}\right) & \mathcal{L}_{c m}^{-1}\left(\mathbf{r}, \mathbf{r}^{\prime}\right) \hat{\alpha}_{m} d_{n}\left(\mathbf{r}, \mathbf{r}^{\prime} ; S_{d}\right) & \mathcal{L}_{c c}^{-1}\left(\mathbf{r}, \mathbf{r}^{\prime}\right) \hat{\alpha}_{c} d\left(\mathbf{r}, \mathbf{r}^{\prime} ; S_{c}\right)
\end{array}\right)
\end{aligned}
$$

where $(18),(22),(23),(31)$ are used. The integral (35) is in fact a global integral defined over the entire surface $S$. It is originally written in terms of the local coordinates $\varphi_{i}$ on the patch $U_{i}$. Afterwards, the partition of unity technique [52] is used to aggregate all the local patches in order to give the total coordinates. The details of this construction are straightforward but rather technical so a summary of the background is relegated to Appendix A. Using that material, the expression (35) can be expanded as

$$
\left[\mathbf{J}_{n}(\mathbf{r})\right]=\sum_{j \in J} \int_{\varphi_{j}\left(U_{j}\right)}\left(\psi_{j}\left(\mathbf{r}^{\prime}\right) \sqrt{\left|g\left(\mathbf{r}^{\prime}\right)\right|}\left[\overline{\mathbf{F}}_{n}\left(\mathbf{r}, \mathbf{r}^{\prime}\right)\right] \cdot\left[\mathbf{V}\left(\mathbf{r}^{\prime}\right)\right]\right) \circ \varphi_{j}^{-1} \mathrm{~d}^{2} x^{\prime}
$$

Here, $g\left(\mathbf{r}^{\prime}\right)$ is the Riemannian metric tensor field on the particular 2-manifold being integrated over, i.e., the surface $S_{c}$ or $S_{d}$. Each of the individual integrals in the RHS of (37) is performed over a local Euclidean patch of the entire surface, namely 
$\varphi\left(U_{i}\right) \subset \mathbb{R}^{2}$, i.e., the coordinate patch corresponding to the the geometric piece $U_{i}$ over which the integration will be numerically preformed. Note that the infinitesimal surface differential element $\mathrm{d}^{2} x^{\prime}$ is mapped by the function $\varphi_{j}^{-1}$ to the global language (intrinsic geometric) representation of points as $\mathbf{r}, \mathbf{r}^{\prime} \in S$. In what follows, all global integrals must be understood in the geometric sense given above and the values obtained are always independent of which coordinate system was used. However, for the sake of brevity, we will not spell out fine-grained expressions like (19) every time an integral over an entire manifold $S_{d}$ or $S_{c}$ is written.

Because all of the media in our system are assumed to be reciprocal, the global operator $\mathcal{L}$ in (17) is itself reciprocal and hence Lorenz reciprocity theorem is valid for our case $[7,9,10,35]$. Using a technical argument identical to the one developed in details in $[1,15]$, it can be shown that the actual current response array $[\mathbf{J}(\mathbf{r})]$ is the limit of the $n$-level approximation $\left[\mathbf{J}_{n}(\mathbf{r})\right]$, that is, we have

$$
[\mathbf{J}(\mathbf{r})]=\lim _{n \rightarrow \infty}\left[\mathbf{J}_{n}(\mathbf{r})\right]=\lim _{n \rightarrow \infty} \int_{S} \mathrm{~d} s^{\prime}\left[\overline{\mathbf{F}}_{n}\left(\mathbf{r}, \mathbf{r}^{\prime}\right)\right] \cdot\left[\mathbf{V}\left(\mathbf{r}^{\prime}\right)\right] .
$$

Since the limit in the RHS can be further pushed inside the integral (the exchange of limit and operators is proved based on reciprocity [15]), it can be concluded that

$$
\left[\overline{\mathbf{F}}\left(\mathbf{r}, \mathbf{r}^{\prime}\right)\right]=\lim _{n \rightarrow \infty}\left[\overline{\mathbf{F}}_{n}\left(\mathbf{r}, \mathbf{r}^{\prime}\right)\right]
$$

which is paramount to saying that the generalized ACGF of the system is the distributional limit of the sequence of $n$-level ordinary tensorial functions $\left[\overline{\mathbf{F}}_{n}\left(\mathbf{r}, \mathbf{r}^{\prime}\right)\right]$. In other words, we may write

$$
\left[\overline{\mathbf{F}}\left(\mathbf{r}, \mathbf{r}^{\prime}\right)\right]: \stackrel{\text { g.f }}{=}\left(\overline{\mathbf{F}}_{1}\left(\mathbf{r}, \mathbf{r}^{\prime}\right) \quad \overline{\mathbf{F}}_{2}\left(\mathbf{r}, \mathbf{r}^{\prime}\right) \quad \cdots \quad \overline{\mathbf{F}}_{n}\left(\mathbf{r}, \mathbf{r}^{\prime}\right) \quad \cdots\right) .
$$

The details of the proof of the above mentioned limit-operator order exchange, already implicit in the statement of distributional approximation (39), are quite lengthy and identical to the PEC case treated in [1] and so will not be given here. $\uparrow$ It now immediately follows from (38) and (39) that

$$
[\mathbf{J}(\mathbf{r})]=\int_{S} \mathrm{~d} s^{\prime}\left[\overline{\mathbf{F}}\left(\mathbf{r}, \mathbf{r}^{\prime}\right)\right] \cdot\left[\mathbf{V}\left(\mathbf{r}^{\prime}\right)\right] .
$$

This is the main relation in the generalized ACGF formalism. If we expand it in details, we find

$$
\begin{aligned}
& \mathbf{J}_{e}(\mathbf{r})=\int_{S_{d}} \mathrm{~d} s^{\prime} \overline{\mathbf{F}}_{e e}\left(\mathbf{r}, \mathbf{r}^{\prime}\right) \cdot \mathbf{E}_{e}^{\mathrm{ex}}\left(\mathbf{r}^{\prime}\right)+\int_{S_{d}} \mathrm{~d} s^{\prime} \overline{\mathbf{F}}_{e m}\left(\mathbf{r}, \mathbf{r}^{\prime}\right) \cdot \mathbf{H}_{m}^{\mathrm{ex}}\left(\mathbf{r}^{\prime}\right) \\
&+\int_{S_{c}} \mathrm{~d} s^{\prime} \overline{\mathbf{F}}_{e c}\left(\mathbf{r}, \mathbf{r}^{\prime}\right) \cdot \mathbf{E}_{c}^{\mathrm{ex}}\left(\mathbf{r}^{\prime}\right), \\
& \mathbf{J}_{m}(\mathbf{r})=\int_{S_{d}} \mathrm{~d} s^{\prime} \overline{\mathbf{F}}_{m e}\left(\mathbf{r}, \mathbf{r}^{\prime}\right) \cdot \mathbf{E}_{e}^{\mathrm{ex}}\left(\mathbf{r}^{\prime}\right)+\int_{S_{d}} \mathrm{~d} s^{\prime} \overline{\mathbf{F}}_{m m}\left(\mathbf{r}, \mathbf{r}^{\prime}\right) \cdot \mathbf{H}_{m}^{\mathrm{ex}}\left(\mathbf{r}^{\prime}\right) \\
&+\int_{S_{c}} \mathrm{~d} s^{\prime} \overline{\mathbf{F}}_{m c}\left(\mathbf{r}, \mathbf{r}^{\prime}\right) \cdot \mathbf{E}_{c}^{\mathrm{ex}}\left(\mathbf{r}^{\prime}\right),
\end{aligned}
$$

I However, the reader should notice that it is not currently known whether the ACGF can be constructed in nonreciprocal systems. 


$$
\begin{array}{r}
\mathbf{J}_{c}(\mathbf{r})=\int_{S_{d}} \mathrm{~d} s^{\prime} \overline{\mathbf{F}}_{c e}\left(\mathbf{r}, \mathbf{r}^{\prime}\right) \cdot \mathbf{E}_{e}^{\mathrm{ex}}\left(\mathbf{r}^{\prime}\right)+\int_{S_{d}} \mathrm{~d} s^{\prime} \overline{\mathbf{F}}_{c m}\left(\mathbf{r}, \mathbf{r}^{\prime}\right) \cdot \mathbf{H}_{m}^{\mathrm{ex}}\left(\mathbf{r}^{\prime}\right) \\
+\int_{S_{c}} \mathrm{~d} s^{\prime} \overline{\mathbf{F}}_{c c}\left(\mathbf{r}, \mathbf{r}^{\prime}\right) \cdot \mathbf{E}_{c}^{\mathrm{ex}}\left(\mathbf{r}^{\prime}\right) .
\end{array}
$$

The relations (41), (42), (43), and (44) provide a complete space-frequency representation of a composite PEC-dielectric system response to external field illuminations $\mathbf{E}^{\mathrm{ex}}(\mathbf{r})$ and $\mathbf{H}^{\mathrm{ex}}(\mathbf{r})$. They are valid for arbitrary inhomogeneous configuration composed of reciprocal domains in which the interfaces can be modeled by orientable smooth 2-manifolds. Further discussion of these final results will be briefly outlined in Sec. 3 .

\subsection{Generalization to Generic PEC-Dielectric Systems}

We end this section by the generalization to the case of arbitrary number of PEC and dielectric domains. The main derivation is identical to the minimal case of one PEC and one dielectric domain developed in details above. We merely expand each surface type into the set-theoretic union of the corresponding sub-surfaces as follows:

$$
S=S_{c} \cup S_{d}, \quad S_{c}=\bigcup_{i=1}^{N_{c}} S_{c}^{i}, \quad S_{d}=\bigcup_{i=1}^{N_{d}} S_{d}^{i}
$$

where $N_{c}$ and $N_{d}$ are the numbers of conducting and dielectric subdomains, respectively. Since each of these sub-surfaces constitutes by itself a smooth Riemannian manifold, the set $S=S_{c} \cup S_{d}$ is also a Riemannian manifold, as well as $S_{c}$ and $S_{d}$ taken individually. Each PEC/dielectric subdomain is enclosed by $S_{c} / S_{d}$, respectively. Furthermore, it is clear that (15) and (16) can be recast into larger arrays with structures

$$
[\mathbf{J}(\mathbf{r})]_{\left(2 N_{e}+N_{c}\right) \times 1}:=\left(\begin{array}{c}
{\left[\mathbf{J}_{e}(\mathbf{r})\right]_{N_{e} \times 1}} \\
{\left[\mathbf{J}_{m}(\mathbf{r})\right]_{N_{e} \times 1}} \\
{\left[\mathbf{J}_{c}(\mathbf{r})\right]_{N_{c} \times 1}}
\end{array}\right),\left[\mathbf{V}^{\mathrm{ex}}(\mathbf{r})\right]_{\left(2 N_{e}+N_{c}\right) \times 1}:=\hat{u}(\mathbf{r}) \times\left(\begin{array}{l}
{\left[\mathbf{E}_{e}^{\mathrm{ex}}(\mathbf{r})\right]_{N_{e} \times 1}} \\
{\left[\mathbf{H}_{m}^{\mathrm{ex}}(\mathbf{r})\right]_{N_{e} \times 1}} \\
{\left[\mathbf{E}_{c}^{\mathrm{ex}}(\mathbf{r})\right]_{N_{c} \times 1}}
\end{array}\right) .
$$

Here, the components of these augmented arrays are

$$
\begin{aligned}
{\left[\mathbf{J}_{e}(\mathbf{r})\right]_{N_{e} \times 1} } & =\left[\mathbf{J}_{e}^{i}(\mathbf{r})\right]_{i=1, \cdots, N_{e}}, \\
{\left[\mathbf{J}_{m}(\mathbf{r})\right]_{N_{e} \times 1} } & =\left[\mathbf{J}_{m}^{i}(\mathbf{r})\right]_{i=1, \cdots, N_{e}}, \\
{\left[\mathbf{J}_{c}(\mathbf{r})\right]_{N_{c} \times 1} } & =\left[\mathbf{J}_{c}^{i}(\mathbf{r})\right]_{i=1, \cdots, N_{c}},
\end{aligned}
$$

where the individual currents with superscript $i$ indexes the currents on the surfaces $S_{c}^{i}$ or $S_{d}^{i}$. Similarly, we have

$$
\begin{gathered}
{\left[\mathbf{E}_{e}^{\mathrm{ex}, i}(\mathbf{r})\right]_{N_{e} \times 1}=\left[\mathbf{E}_{e}^{\mathrm{ex}, i}(\mathbf{r})\right]_{i=1, \cdots, N_{e}},} \\
{\left[\mathbf{H}_{m}^{\mathrm{ex}, i}(\mathbf{r})\right]_{N_{e} \times 1}=\left[\mathbf{H}_{m}^{\mathrm{ex}, i}(\mathbf{r})\right]_{i=1, \cdots, N_{e}},} \\
{\left[\mathbf{E}_{c}^{\mathrm{ex}, i}(\mathbf{r})\right]_{N_{c} \times 1}=\left[\mathbf{E}_{c}^{\mathrm{ex}, i}(\mathbf{r})\right]_{i=1, \cdots, N_{c}}}
\end{gathered}
$$


The pattern is now clear. We will not give the analog to all intermediate relations, but mention the generalized ACGF augmented array, which in the most generic scenario acquires the form

$$
\begin{aligned}
& {\left[\overline{\mathbf{F}}\left(\mathbf{r}, \mathbf{r}^{\prime}\right)\right]_{\left(2 N_{e}+N_{c}\right) \times\left(2 N_{e}+N_{c}\right)}} \\
& \quad=\left(\begin{array}{lll}
{\left[\overline{\mathbf{F}}_{e e}\left(\mathbf{r}, \mathbf{r}^{\prime}\right)\right]_{N_{e} \times N_{e}}} & {\left[\overline{\mathbf{F}}_{e m}\left(\mathbf{r}, \mathbf{r}^{\prime}\right)\right]_{N_{e} \times N_{e}}} & {\left[\overline{\mathbf{F}}_{e c}\left(\mathbf{r}, \mathbf{r}^{\prime}\right)\right]_{N_{e} \times N_{c}}} \\
{\left[\overline{\mathbf{F}}_{m e}\left(\mathbf{r}, \mathbf{r}^{\prime}\right)\right]_{N_{e} \times N_{e}}} & {\left[\overline{\mathbf{F}}_{m m}\left(\mathbf{r}, \mathbf{r}^{\prime}\right)\right]_{N_{e} \times N_{e}}} & {\left[\overline{\mathbf{F}}_{m c}\left(\mathbf{r}, \mathbf{r}^{\prime}\right)\right]_{N_{e} \times N_{c}}} \\
{\left[\overline{\mathbf{F}}_{c e}\left(\mathbf{r}, \mathbf{r}^{\prime}\right)\right]_{N_{c} \times N_{e}}} & {\left[\overline{\mathbf{F}}_{c m}\left(\mathbf{r}, \mathbf{r}^{\prime}\right)\right]_{N_{c} \times N_{e}}} & {\left[\overline{\mathbf{F}}_{c c}\left(\mathbf{r}, \mathbf{r}^{\prime}\right)\right]_{N_{c} \times N_{c}}}
\end{array}\right) .
\end{aligned}
$$

Here, each element of the augmented generalized ACGF array $\left[\overline{\mathbf{F}}\left(\mathbf{r}, \mathbf{r}^{\prime}\right)\right]$ is written as $\overline{\mathbf{F}}_{a b}^{i j}$, itself a tensor on the appropriate 2-manifold, with data structure conforming to

$$
\left[\overline{\mathbf{F}}\left(\mathbf{r}, \mathbf{r}^{\prime}\right)\right]_{\left(2 N_{e}+N_{c}\right) \times\left(2 N_{e}+N_{c}\right)}=\left[\overline{\mathbf{F}}_{a b}^{i j}\left(\mathbf{r}, \mathbf{r}^{\prime}\right)\right]_{a, b \in\{e, m, c\} ; i, j \in\left\{1, \cdots, \max \left(N_{c}, N_{e}\right)\right\}} .
$$

The detailed forms for all these sub-components are listed in Appendix C, which must be consulted in order to correctly implement the generalized ACGF tensor array $\left[\overline{\mathbf{F}}\left(\mathbf{r}, \mathbf{r}^{\prime}\right)\right]$. Note further that each tensor sub-component $\overline{\mathbf{F}}_{a b}^{i j}$ is itself expressible in terms of one of the formulas listed in Appendix B. Consequently, combining the expressions in both the latter Appendix with those found in Appendix C, the global generalized ACGF tensor array can be ultimately traced back to individual scalar components that might be implemented numerically or measured in the lab. Based on this notation, the final generalized ACGF relation becomes

$$
[\mathbf{J}(\mathbf{r})]_{\left(2 N_{e}+N_{c}\right) \times 1}=\int_{S} \mathrm{~d} s^{\prime}\left[\overline{\mathbf{F}}\left(\mathbf{r}, \mathbf{r}^{\prime}\right)\right]_{\left(2 N_{e}+N_{c}\right) \times\left(2 N_{e}+N_{c}\right)} \cdot\left[\mathbf{V}\left(\mathbf{r}^{\prime}\right)\right]_{\left(2 N_{e}+N_{c}\right) \times 1} .
$$

In details, the various currents flowing on the sub-surfaces $S_{c}^{i}$ and $S_{d}^{i}$ an be expressed in terms of all other excitation field through the relations

$$
\begin{aligned}
& \mathbf{J}_{e}^{i}(\mathbf{r})=\sum_{j=1}^{N_{e}} \int_{S_{d}^{j}} \mathrm{~d} s^{\prime} \overline{\mathbf{F}}_{e e}^{i j}\left(\mathbf{r}, \mathbf{r}^{\prime}\right) \cdot \mathbf{E}_{e}^{\mathrm{ex}, j}\left(\mathbf{r}^{\prime}\right)+\sum_{j=1}^{N_{e}} \int_{S_{d}^{j}} \mathrm{~d} s^{\prime} \overline{\mathbf{F}}_{e m}^{i j}\left(\mathbf{r}, \mathbf{r}^{\prime}\right) \cdot \mathbf{E}_{m}^{\mathrm{ex}, j}\left(\mathbf{r}^{\prime}\right) \\
&+\sum_{j=1}^{N_{c}} \int_{S_{c}^{j}} \mathrm{~d} s^{\prime} \overline{\mathbf{F}}_{e c}^{i j}\left(\mathbf{r}, \mathbf{r}^{\prime}\right) \cdot \mathbf{E}_{c}^{\mathrm{ex}, j}\left(\mathbf{r}^{\prime}\right), \\
& \mathbf{J}_{m}^{i}(\mathbf{r})=\sum_{j=1}^{N_{e}} \int_{S_{\mathrm{d}}^{j}} d^{3} r^{\prime} \overline{\mathbf{F}}_{m e}^{i j}\left(\mathbf{r}, \mathbf{r}^{\prime}\right) \cdot \mathbf{E}_{e}^{\mathrm{ex}, j}\left(\mathbf{r}^{\prime}\right)+\sum_{j=1}^{N_{e}} \int_{S_{d}^{j}} \mathrm{~d} s^{\prime} \overline{\mathbf{F}}_{m m}\left(\mathbf{r}, \mathbf{r}^{\prime}\right) \cdot \mathbf{E}_{m}^{\mathrm{ex}, j}\left(\mathbf{r}^{\prime}\right) \\
& \\
&+\sum_{j=1}^{N_{c}} \int_{S_{c}^{j}} \mathrm{~d} s^{\prime} \overline{\mathbf{F}}_{m c}\left(\mathbf{r}, \mathbf{r}^{\prime}\right) \cdot \mathbf{E}_{c}^{\mathrm{ex}, j}\left(\mathbf{r}^{\prime}\right),
\end{aligned}
$$




$$
\begin{aligned}
\mathbf{J}_{c}^{i}(\mathbf{r})=\sum_{j=1}^{N_{e}} \int_{S_{d}^{j}} \mathrm{~d} s^{\prime} \overline{\mathbf{F}}_{c e}^{i j}\left(\mathbf{r}, \mathbf{r}^{\prime}\right) \cdot \mathbf{E}_{e}^{\mathrm{ex}, j}\left(\mathbf{r}^{\prime}\right)+\sum_{j=1}^{N_{e}} \int_{S_{d}^{j}} \mathrm{~d} s^{\prime} \overline{\mathbf{F}}_{c m}^{i j}\left(\mathbf{r}, \mathbf{r}^{\prime}\right) \cdot \mathbf{E}_{m}^{\mathrm{ex}, j}\left(\mathbf{r}^{\prime}\right) \\
\\
+\sum_{j=1}^{N_{c}} \int_{S_{c}^{j}} \mathrm{~d} s^{\prime} \overline{\mathbf{F}}_{c c}^{i j}\left(\mathbf{r}, \mathbf{r}^{\prime}\right) \cdot \mathbf{E}_{c}^{\mathrm{ex}, j}\left(\mathbf{r}^{\prime}\right) .
\end{aligned}
$$

The three relations (52)-(44) represent the most general form possible of the current Green's function method in generic composite PEC-dielectric systems and is stated and derived here for the first time but under the assumption that the media composing the system are reciprocal.

\section{Discussion of the Results and Further General Remarks}

We provide in this last section a set of general observations and remarks with the goal to situate the fundamental mathematical formulation presented above within the general context of both theory and applications. First, we would like to compare the coupled PEC-dielectric formalism with the previously published PEC ACGF method [1]. It is immediately noticed that while there are several similarities, for example the formal structure of the superposition integral (51), there are also fundamental differences. The most important, in our view, is the considerable increase in complexity due to the need to take into account the magnetic field interaction. Indeed, the magnetic field will contribute at every point in the surface $S_{d}$, and even the conduction current $\mathbf{J}_{c}^{i}(\mathbf{r})$, where $\mathbf{r} \in S_{c}^{i}$, will receive contribution from not only the electric fields at all other surfaces $s_{d}^{j}$, but also the magnetic fields there. This curious situation shows that mixed PEC-dielectric systems are inherently more complex. Mutual coupling exists between every two domains, and this coupling is taken into account through coupling Green's functions of the form $\overline{\mathbf{F}}_{a b}^{i j}\left(\mathbf{r}, \mathbf{r}^{\prime}\right)$.

Next, we note not only the formal complexity of the generalized ACGF method, but also anticipated considerable increase in computational demands. This is already clear in the generic formulas treated in Sec. 2.3 where each tensor was replaced by an array. In fact, the generalized ACGF of a generic composite PEC-dielectric appearing in (49) is an $\left(2 N_{e}+N_{c}\right) \times\left(2 N_{e}+N_{c}\right)$ square array, where $N_{e}$ and $N_{c}$ are the total numbers of dielectric and PEC sub-domains, numbers that can easily increase with complicated antenna types like multi-layered microstrip antennas [53] or multiplescattering systems $[9,10]$. Now every "element" of this array, i.e., the generic term

$\overline{\mathbf{F}}_{a b}^{i j}\left(\mathbf{r}, \mathbf{r}^{\prime}\right)$, is itself a tensor, or simply a dyad, with representation in a chosen (local) coordinate system on the 2-manifold on which this dyad is def ind giving rise to a $2 \times 2$ matrix as per the detailed expressions in Appendix B and Appendix C. Therefore, the generalized ACGF array is effectively a rather complicated and involved matrix of $\left(4 N_{e}+2 N_{c}\right) \times\left(4 N_{e}+2 N_{c}\right)$ scalar functions. This matrix is coordinate dependent in the sense that the its entries' exact numerical values depend on the coordinate system under consideration. However, since we use local charts (intrinsic manifold language), the final results, the current induced on the surface $S$ after interaction with incident excitation fields, as given by the integral (51), is independent of the coordinate system. In fact, a concrete calculus for transforming ACGF tensors was derived in [1] and can be also developed here with minor changes. Since the idea is the same but the details are lengthy, this method will not be given here but the reader need to be aware that 
the ACGF as constructed here is like all tensors a geometric quantity, i.e., not attached to a specific coordinate system. ${ }^{+}$

Few remarks on the range of validity of the derived formalism. It is important to note that technically the existence of the generalized ACGF depends crucially on the assumption that the system is reciprocal. This, however, is a character in our particular construction. It is currently an open question whether a different approach can be applied to construct the ACGF for nonreciprocal systems. It is in general believed that Green's functions and reciprocity are intimately related to each other, but since our constructive method is not unique, we prefer to leave the question of whether ACGFs exists in nonreciprocal radiating systems for future investigations. Another restriction that must be noted is that our use of the surface equivalence theorem to construct the generalized ACGF formalism formulates the problem exclusively in terms of surface currents. It is currently an open question whether the ACGF can also be constructed using volume integral equations, leading to to volume current response. That will lead to substantial modification (if the formulation indeed is possible) since the use of Riemannian geometry will become less warranted as in our case where surface integral equations has been selected as the ultimate foundations of the method right from the start.

We finally say few words about the numerical implementation of the ACGF formalism. Strictly speaking, the ACGF technique was originally introduced essentially as an alternative numerical method in electromagnetics, with applications to the analysis of wire antennas [30]. After developing some of the theoretical foundations in $[14,15]$, a direct computational technique using the Method of Moment (MoM) was proposed in [16], which is similar to [30]. The basic idea is to use MoM with specialized mesh to model an increasing number of $n$-level distributional approximations of the ACGF, namely the $\overline{\overline{\mathbf{F}}}_{n}$ appearing in (28). This idea was investigated numerically in $[24,54]$ and experimentally in [18] for mixed PEC-dielectric type antennas (strongly coupled microstrip antennas backed by dielectric substrate and ground plane), without, however, explicit mention or derivation of the integal formulas (51). Afterwards, this MoM-based approach has been further systematized and integrated with more applications, e.g., see [1]. However, it is very important to note that the MoM-based approach to the ACGF method is not the only option available as we will discuss shortly. In fact, the ACGF, being more fundamental than the discrete mesh approximation upon which all numerical methods like the MoM are based, can be even measured directly in the lab, though to the best of our knowledge this has not been accomplished yet. The problem with MoM-based estimation is that it is very expensive and may lead to convergence problems. Indeed, each $n$-level approximation $\overline{\mathbf{F}}_{n}\left(\mathbf{r}, \mathbf{r}^{\prime}\right)$ requires a dense, most often nonuniform, MoM discretization mesh in order to properly model the physics of the progressively narrowing excitation $d_{n}\left(\mathbf{r}, \mathbf{r}^{\prime}\right)$ that approximate in turn the surface delta function as per (18). Since the MoM is well known to suffer from convergence problems when the matrix to be inverted becomes denser [55], this direct approach via the distributional approximation relation (39) may become prohibitively expensive or even simply not convergent if the original (exact) limit is replaced by a MoM-based operator approximation. Unfortunately, not much has been published on the pure numerical issue of convergence rate and speed

+ In practical applications to complex antennas that involve closed surfaces this is important since full-wave numerical simulators tend to use local (geometric) coordinate systems, e.g., the curvilinear coordinates of the old Gaussian theory of 2-surfaces, e.g., see [39, 40]. 
in the MoM-based approach to the ACGF method.

It is interesting to note that in recent years two alternative techniques have been proposed to estimate the ACGF. One technique is in fact as old as the ACGF method itself, and was already used in the earliest publications [30]. This is the proposal to use the spatial singularity expansion method (S-SEM) to approximate the ACGF as a finite series of complex exponentials, in a way that resembles the well known eigenfunction expansion method or singularity expansion method in the time domain [56]. While the spatial SEM itself was developed systematically only very recently in [57], it had been already applied to estimate the ACGF of printed antennas in [58-60]. This method rely on measuring some near- or far- field and then using machine learning to perform inverse source modeling to determine the unknown current on the radiator using the S-SEM expansion as learning model to be trained with available data $[61,62]$. The second new method alternative to MoM-based approximation is the infinitesimal dipole model (IDM) approach, which is referred to here as the ACGF-IDM framework. The IDM method is a very general strategy in computational electromagnetics aiming at replacing a complex radiating current by an equivalent small number of concentrated Dirac-like sources [63]. The method intersects a large number of applications in areas as diverse as imaging, geo-sensing, radars, wireless communications, tomography, and others. ${ }^{*}$ It was shown recently that a combination of the ACGF and IDM methods can effectively model complex radiating systems using measured far-field data [23]. This approach was applied there with experimental and numerical results to conformal microstrip antenna arrays, which also involve composite PEC-dielectric objects. Finally, we mention also some hybrid methods involving a combination of hybrid ACGF and physical optics approach as in $[21,22]$. To summarize, aside from direct lab measurement of the ACGF itself (not realized yet), so far four different numerical methods have been used to implement the ACGF technique:

(i) The direct (dense) full-wave method approximation (most often using the MoM).

(ii) The ACGF-SEM method (based on measured field data).

(iii) The ACGF-IDM method (based on measured field data).

(iv) Hybrid methods combining the full-wave numerical approximation approach to the ACGF with other approximations such as ray tracing, physical optics, etc.

The state of research on the computational efficiency of the proposed ACGF formalism as an alternative method in CEM is still evolving and we hope more work on this will be published in the present new decade.

\section{Conclusion}

Starting from surface integral equations, we provided an extensive rigorous mathematical investigation into the foundations and derivation of the main tenets of the antenna current Green's function (ACGF) formalism for coupled PEC-dielectric systems, filling an existing gap in the literature where most investigations using the ACGF method has focused so far on numerical and experimental aspects. In fact, previously only the perfect-electric conducting (PEC) case was investigated mathematically at the fundamental level. It was found that in contrast to the PEC ACGF formalism, including dielectric domains with possible magnetic properties

* See the literature quoted in [64] for some recent survey on the IDM method. 
brings both electric and magnetic fields into the picture, i.e., not only the electric field as in the PEC case. Also, both electric and magnetic currents are introduced at dielectric interfaces and the needed general surface integral relations in terms of the ACGF (Green's kernel) were derived. The construction of the mixed PEC-dielectric ACGF gave rise to a higher-order tensor array, considerably more complicated than the PEC's tensor. Our derivation depends crucially on assuming reciprocity in additional to linearity and it is currently unknown if the ACGF can be constructed for nonreciprocal systems or whether the ACGF can be constructed based on volume integral equations.

\section{Appendix A. Coordinate-Independent Extension of Integrals from Local to Global Manifold Domains}

Let $(M, g)$ be an $n$-dimensional riemannian manifold with topological manifold $M$ and metric tensor field $g$. An atlas on $M$ is given by $U_{i}, \varphi_{i}, i \in I$. We say that $\left[U_{j}, \varphi_{j}(\mathbf{r}), \psi_{j}(\mathbf{r})\right], j \in J, \mathbf{r} \in M$, is a partition of unity subordinate to $U_{i}, \varphi_{i}, i \in I$, when:

(i) The collection of functions $\psi_{j}: M \rightarrow \mathbb{R}$ on $M$ constitutes a smooth partition of unity subordinate to the covering $U_{i}, i \in I$.

(ii) $\left(U_{j}, \varphi_{j}\right), j \in J$, in itself constitutes an atlas on $M$.

(iii) For any $j \in J$, the support of of $\psi_{j}(\mathbf{r})$ is contained within $U_{j}$, i.e., $\operatorname{supp}\left(\psi_{j}\right) \subset U_{j}$.

It can be shown that for any atlas there exists a partition of unity subordinate to it in the sense given above [52]. Now, since $x=\varphi_{j}(\mathbf{r})$ is in $\mathbb{R}^{n}$, then we can immediately invoke the classic Lebesgue measure on Euclidean spaces to write integrals with respect to $x$, which will be in the form $\int \mathrm{d}^{n} x$. We then define Riemannian measure integral on a given function $f: M \rightarrow \mathbb{R}$ by the formula

$$
\int_{M} \mathrm{~d} v f:=\sum_{j \in J} \int_{\varphi_{j}\left(U_{j}\right)}\left(\psi_{j} \sqrt{|g|} f\right) \circ \varphi_{j}^{-1} \mathrm{~d}^{n} x
$$

where $|g|$ is the determinant of the metric tensor $g$ when its components are expressed in terms of the local coordinate system of the $j$ th patch. In this way, the integral defined globally over the entire manifold is expanded into the aggregation of smaller integrals over the local patches $U_{j}$ which when pieced up together give back the total manifold $M$. We note that for definition to work, we assume that the integrated function has a compact support, a condition automatically satisfied in our case because all manifolds dealt with in this paper, namely $S_{c}$ and $S_{d}$ are compact. Finally, it can be shown that the construction (A.1) is independent of which coordinate system was used [52]. In our case, the surface definition corresponding to $n=2$ is the only one needed.

\section{Appendix B. Tensorial Expansions for all Global Generalized ACGF Components}

$$
\overline{\mathbf{F}}_{e e}\left(\mathbf{r}, \mathbf{r}^{\prime}\right)=\left\{\begin{array}{cl}
\sum_{i, j=1}^{2} \hat{\alpha}_{i}(\mathbf{r}) \hat{\alpha}_{j}\left(\mathbf{r}^{\prime}\right) F_{e e}^{i j}\left(\mathbf{r}, \mathbf{r}^{\prime}\right), & \mathbf{r}^{\prime} \in S_{d}, \mathbf{r} \in S_{d} \\
0, & \text { elsewhere }
\end{array}\right.
$$




$$
\begin{gathered}
\overline{\mathbf{F}}_{e m}\left(\mathbf{r}, \mathbf{r}^{\prime}\right)=\left\{\begin{array}{cl}
\sum_{i, j=1}^{2} \hat{\alpha}_{i}(\mathbf{r}) \hat{\alpha}_{j}\left(\mathbf{r}^{\prime}\right) F_{e m}^{i j}\left(\mathbf{r}, \mathbf{r}^{\prime}\right), & \mathbf{r}^{\prime} \in S_{d}, \mathbf{r} \in S_{d}, \\
0, & \text { elsewhere. }
\end{array}\right. \\
\overline{\mathbf{F}}_{e c}\left(\mathbf{r}, \mathbf{r}^{\prime}\right)=\left\{\begin{array}{cl}
\sum_{i, j=1}^{2} \hat{\alpha}_{i}(\mathbf{r}) \hat{\alpha}_{j}\left(\mathbf{r}^{\prime}\right) F_{e c}^{i j}\left(\mathbf{r}, \mathbf{r}^{\prime}\right), & \mathbf{r}^{\prime} \in S_{c}, \mathbf{r} \in S_{d}, \\
0, & \text { elsewhere. }
\end{array}\right. \\
\overline{\mathbf{F}}_{m e}\left(\mathbf{r}, \mathbf{r}^{\prime}\right)=\left\{\begin{array}{cl}
\sum_{i, j=1}^{2} \hat{\alpha}_{i}(\mathbf{r}) \hat{\alpha}_{j}\left(\mathbf{r}^{\prime}\right) F_{m e}^{i j}\left(\mathbf{r}, \mathbf{r}^{\prime}\right), & \mathbf{r}^{\prime} \in S_{d}, \mathbf{r} \in S_{d}, \\
0, & \text { elsewhere. }
\end{array}\right. \\
\overline{\mathbf{F}}_{m m}\left(\mathbf{r}, \mathbf{r}^{\prime}\right)=\left\{\begin{array}{cl}
\sum_{i, j=1}^{2} \hat{\alpha}_{i}(\mathbf{r}) \hat{\alpha}_{j}\left(\mathbf{r}^{\prime}\right) F_{m m}^{i j}\left(\mathbf{r}, \mathbf{r}^{\prime}\right), & \mathbf{r}^{\prime} \in S_{d}, \mathbf{r} \in S_{d} \\
0, & \text { elsewhere. }
\end{array}\right. \\
\overline{\mathbf{F}}_{c e}\left(\mathbf{r}, \mathbf{r}^{\prime}\right)=\left\{\begin{array}{cl}
\sum_{i, j=1}^{2} \hat{\alpha}_{i}(\mathbf{r}) \hat{\alpha}_{j}\left(\mathbf{r}^{\prime}\right) F_{c e}^{i j}\left(\mathbf{r}, \mathbf{r}^{\prime}\right), & \mathbf{r}^{\prime} \in S_{d}, \mathbf{r} \in S_{c} \\
0, & \text { elsewhere. } \\
0, & \mathbf{r}^{\prime} \in S_{c}, \mathbf{r} \in S_{d},
\end{array}\right. \\
\overline{\mathbf{F}}_{c m}\left(\mathbf{r}, \mathbf{r}^{\prime}\right)=\left\{\begin{array}{cl}
\sum_{i, j=1}^{2} \hat{\alpha}_{i}(\mathbf{r}) \hat{\alpha}_{j}\left(\mathbf{r}^{\prime}\right) F_{m c}^{i j}\left(\mathbf{r}, \mathbf{r}_{i}^{\prime}(\mathbf{r}) \hat{\alpha}_{j}\left(\mathbf{r}^{\prime}\right) F_{c m}^{i j}\left(\mathbf{r}, \mathbf{r}^{\prime}\right),\right. & \mathbf{r}^{\prime} \in S_{d}, \mathbf{r} \in S_{c}, \\
0, & \text { elsewhere. }
\end{array}\right. \\
\overline{\mathbf{F}}_{c c}\left(\mathbf{r}, \mathbf{r}^{\prime}\right)=\left\{\begin{array}{cl}
\sum_{i, j=1}^{2} \hat{\alpha}_{i}(\mathbf{r}) \hat{\alpha}_{j}\left(\mathbf{r}^{\prime}\right) F_{c c}^{i j}\left(\mathbf{r}, \mathbf{r}^{\prime}\right), & \mathbf{r}^{\prime} \in S_{c}, \mathbf{r} \in S_{c} \\
0, & \text { elsewhere. }
\end{array}\right.
\end{gathered}
$$

Appendix C. Structure of the Generalized ACGF Array Components for Multiple Coupled PEC-Dielectric Systems

$$
\begin{gathered}
{\left[\overline{\mathbf{F}}_{e e}\left(\mathbf{r}, \mathbf{r}^{\prime}\right)\right]_{N_{e} \times N_{e}}=\left[\overline{\mathbf{F}}_{e e}^{i j}\left(\mathbf{r}, \mathbf{r}^{\prime}\right)\right]_{i, j=1, \cdots, N_{e}},} \\
{\left[\overline{\mathbf{F}}_{m e}\left(\mathbf{r}, \mathbf{r}^{\prime}\right)\right]_{N_{e} \times N_{e}}=\left[\overline{\mathbf{F}}_{m e}^{i j}\left(\mathbf{r}, \mathbf{r}^{\prime}\right)\right]_{i, j=1, \cdots, N_{e}},} \\
{\left[\overline{\mathbf{F}}_{e m}\left(\mathbf{r}, \mathbf{r}^{\prime}\right)\right]_{N_{e} \times N_{e}}=\left[\overline{\mathbf{F}}_{e m}^{i j}\left(\mathbf{r}, \mathbf{r}^{\prime}\right)\right]_{i, j=1, \cdots, N_{e}},} \\
{\left[\overline{\mathbf{F}}_{m m}\left(\mathbf{r}, \mathbf{r}^{\prime}\right)\right]_{N_{e} \times N_{e}}=\left[\overline{\mathbf{F}}_{m m}^{i j}\left(\mathbf{r}, \mathbf{r}^{\prime}\right)\right]_{i, j=1, \cdots, N_{e}},} \\
{\left[\overline{\mathbf{F}}_{e c}\left(\mathbf{r}, \mathbf{r}^{\prime}\right)\right]_{N_{e} \times N_{c}}=\left[\overline{\mathbf{F}}_{e c}^{i j}\left(\mathbf{r}, \mathbf{r}^{\prime}\right)\right]_{i=1, \cdots, N_{e} ; j=1, \cdots, N_{c}},} \\
{\left[\overline{\mathbf{F}}_{c e}\left(\mathbf{r}, \mathbf{r}^{\prime}\right)\right]_{N_{c} \times N_{e}}=\left[\overline{\mathbf{F}}_{c e}^{i j}\left(\mathbf{r}, \mathbf{r}^{\prime}\right)\right]_{i=1, \cdots, N_{c} ; j=1, \cdots, N_{e}},} \\
{\left[\overline{\mathbf{F}}_{c m}\left(\mathbf{r}, \mathbf{r}^{\prime}\right)\right]_{N_{c} \times N_{e}}=\left[\overline{\mathbf{F}}_{c m}^{i j}\left(\mathbf{r}, \mathbf{r}^{\prime}\right)\right]_{i=1, \cdots, N_{c} ; j=1, \cdots, N_{e}},} \\
{\left[\overline{\mathbf{F}}_{m c}\left(\mathbf{r}, \mathbf{r}^{\prime}\right)\right]_{N_{e} \times N_{c}}=\left[\overline{\mathbf{F}}_{m c}^{i j}\left(\mathbf{r}, \mathbf{r}^{\prime}\right)\right]_{i=1, \cdots, N_{e} ; j=1, \cdots, N_{c}},} \\
{\left[\overline{\mathbf{F}}_{c c}\left(\mathbf{r}, \mathbf{r}^{\prime}\right)\right]_{N_{c} \times N_{c}}=\left[\overline{\mathbf{F}}_{c c}^{i j}\left(\mathbf{r}, \mathbf{r}^{\prime}\right)\right]_{i, j=1, \cdots, N_{c}}}
\end{gathered}
$$




\section{References}

[1] S. Mikki and Y. Antar, New Foundations for Applied Electromagnetics: The Spatial Structure of Fields. London: Artech House, 2016.

[2] S. A. Schelkunoff, A Mathematical Theory of Linear Arrays. The Bell System Technical Journal, 1943, vol. 22 .

[3] S. A. Schelkunoff and H. T. Friss, Antennas: Theory and practice. New York; Chapman \& Hall: London, 1952.

[4] K. Cho, Optical response of nanostructures: microscopic nonlocal theory. Berlin New York: Springer, 2003.

[5] O. Keller, Quantum Theory of Near-Field Electrodynamics. Berlin New York: Springer, 2011.

[6] S. Mikki and A. Kishk, "A symmetry-based formalism for the electrodynamics of nanotubes," Progress In Electromagnetics Research, vol. 86, pp. 111-134, 2008.

[7] J. Schwinger et al., Classical electrodynamics. Reading, Mass: Perseus Books, 1998.

[8] J. Jackson, Classical electrodynamics. New York: Wiley, 1999.

[9] L. Felsen, Radiation and Scattering of Waves. Piscataway, NJ: IEEE Press, 1994.

[10] W. C. Chew, Waves and Fields in Inhomogenous Media. Wiley-IEEE, 1999.

[11] U. Jentschura, Advanced classical electrodynamics: green functions, regularizations, multipole decompositions. New Jersey: World Scientific, 2017.

[12] C.-T. Tai, Dyadic green functions in electromagnetic theory. Piscataway, NJ: IEEE Press, 1994.

[13] S. A. Schelkunoff, "Theory of antennas of arbitrary size and shape," Proceedings of the IEEE, vol. 72, no. 9, pp. 1165-1190, Sep. 1984.

[14] S. Mikki and Y. Antar, "On the Fundamental Relationship Between the Transmitting and Receiving Modes of General Antenna Systems: A New Approach," IEEE Antennas and Wireless Propagation Letters, vol. 11, pp. 232-235, 2012.

[15] — - "The antenna current Green's function formalism-Part I," IEEE Trans. Antennas Propagat, vol. 9, pp. 4493-4504, September 2013.

[16] — - "The antenna current Green's function formalism-Part II," IEEE Trans. Antennas Propagat, vol. 9, pp. 4505-4519, September 2013.

[17] — "A rigorous approach to mutual coupling in general antenna systems through perturbation theory," IEEE Antennas and Wireless Communication Letters, vol. 14, pp. 115-118, 2015.

[18] S. Henault, S. K. Podilchak, S. Mikki, and Y. Antar, "A Methodology for Mutual Coupling Estimation and Compensation in Antennas," IEEE Transactions on Antennas and Propagation, vol. 61, no. 3, pp. 1119-1131, March 2013.

[19] A. Alzahed, S. Mikki, and Y. Antar, "Design of nonlinear mutual coupling operator for antenna arrays using a novel ACGF-deep-learning technology," in International Union of Radio Science General Assembly \& Scientific (URSI) Symposium, Montreal, 2017.

[20] - "Nonlinear mutual coupling compensation operator design using a novel electromagnetic machine learning paradigm," IEEE Antennas and Wireless Propagation Letters, vol. 18, no. 5, pp. 861-865, 2019.

[21] Y.-D. Kim, H.-J. Kim, K.-U. Bae, J.-H. Park, and N.-H. Myung, "A hybrid UTD-ACGF technique for DOA finding of receiving antenna array on complex environment," IEEE Transactions on Antennas and Propagation, vol. 63, p. 11, 2015.

[22] Y.-D. Kim, D.-W. Yi, S.-J. Yang, H. Chae, J.-W. Yu, and N.-H. Myung, "Beam pattern analysis of antenna array on complex platform using AEP method based on hybrid UTD-ACGF technique," IEEE Transactions on Antennas and Propagation, vol. 65, p. 3, 2017.

[23] S. Yang, Y. Kim, H. Jo, and N. Myung, "Alternative method for obtaining antenna current Green's function based on infinitesimal dipole modeling," IEEE Transactions on Antennas and Propagation, vol. 67, no. 4, pp. 2583-2590, April 2019.

[24] S. Mikki and Y. Antar, "Analysis of generic near-field interactions using the antenna current Green's function," Progress of Electromagnetic Research C (PIER C), vol. 59, pp. 1-9, 2015.

[25] A. Hanoon and S. Mikki, "Bandwidth-enhancement of digital communication systems employing narrowband antennas: A novel electromagnetic OFDM approach," in 2017 IEEE International Symposium on Antennas and Propagation USNC/URSI National Radio Science Meeting, July 2017, pp. 527-528.

[26] S. Mikki, A. Hanoon, J. Aulin, and Y. Antar, "The time-dependent ACGF with applications to M-ary digital communication systems," in The 11th European Conference on Antennas and Propagation (EuCap 2017), 2017, pp. 19-24.

[27] S. Mikki, "The antenna spacetime system theory of wireless communications," Proceedings of the Royal Society A: Mathematical, Physical and Engineering Sciences, April 2019.

[28] D. Kahn, Introduction to global analysis. Mineola, N.Y: Dover Publications, 2007. 
[29] I. Agricola, Global analysis: differential forms in analysis, geometry, and physics. Providence, R.I: American Mathematical Society, 2002.

[30] S. Mikki and Y. M. Antar, "Analysis of electromagnetic interactions in antenna arrays using the antenna current Green's function method," in Proceedings of IEEE APS-URSI International Symposium, 2011, pp. 3-8.

[31] O. D. Kellogg, Foundations of potential theory. Berlin: J. Springer, 1929.

[32] D. Colton and R. Kress, Inverse acoustic and electromagnetic scattering theory. Cham: Springer, 2019.

[33] J.-C. Nedelec, Acoustic and electromagnetic equations: integral representations for harmonic problems. New York: Springer, 2001.

[34] v. J. Bladel, Electromagnetic fields. Wiley-Intersience, IEEE, 2007.

[35] W. Geyi, Foundations of Applied Electrodynamics. Chichester, West Sussex Hoboken, N.J: Wiley, 2010.

[36] A. E. H. Love, "The integration of the equations of propagation of electric waves," Philosophical Transactions of the Royal Society of London. Series A, Containing Papers of a Mathematical or Physical Character, vol. 197, pp. 1-45, 1901.

[37] S. A. Schelkunoff, "Some equivalence theorems of electromagnetics and their application to radiation problems," The Bell System Technical Journal, vol. 15, no. 1, pp. 92-112, Jan 1936.

[38] W. Chew, M. S. Tong, and B. Hu, Integral equation methods for electromagnetic and elastic waves. San Rafael, Calif: Morgan \& Claypool Publishers, 2009.

[39] W. Chew, J.-M. Jin, E. Michielssen, and J. Song-(editors), Fast and efficient algorithms in computational electromagnetics. Boston: Artech House, 2001.

[40] B. M. Kolundzija and A. R. Djordjevic, Electromagnetic Modeling of Composite Metallic and Dielectric Structures. Artech House, 2003.

[41] K. Warnick and W. Chew, Numerical analysis for electromagnetic integral equations. Boston: Artech House, 2008.

[42] E. Zeidler, Quantum field theory I: Basics in Mathematics and Physics. Springer, 2009.

[43] E. Arvas, A. Rahhal Arabi, A. Sadigh, and S. M. Rao, "Scattering from multiple conducting and dielectric bodies of arbitrary shape," IEEE Antennas and Propagation Magazine, vol. 33, no. 2, pp. 29-36, April 1991.

[44] L. N. Medgyesi-Mitschang, J. M. Putnam, and M. B. Gedera, "Generalized method of moments for three-dimensional penetrable scatterers," J. Opt. Soc. Am. A, vol. 11, no. 4, pp. 13831398, Apr 1994.

[45] P. Yla-Oijala, M. Taskinen, and J. Sarvas, "Surface integral equation method for general composite metallic and dielectric structures with junctions," Progress In Electromagnetics Research, vol. 52, pp. 81-108, 2005.

[46] W. Appel, Mathematics for physics and physicists. Princeton, N.J: Princeton University Press, 2007.

[47] I. Gelfand and G. Shilov, Generalized functions: Volume 1. Providence, Rhode Island: American Mathematical Society AMS Chelsea Publishing, 2016.

[48] E. Zeidler, Quantum field theory II: Quantum Electrodynamics. Springer, 2006.

[49] S. Hassani, Mathematical physics: a modern introduction to its foundations. Cham: Springer, 2013.

[50] I. Gelfand and G. Shilov, Generalized functions: Volume 2. Providence, Rhode Island: American Mathematical Society AMS Chelsea Publishing, 2016.

[51] I. Gelfand and N. Vilenkin, Generalized functions: Volume 4. New York London: Academic Press, 1964.

[52] J. Lee, Introduction to smooth manifolds. New York London: Springer, 2012.

[53] H. Schantz, The art and science of ultrawideband antennas. Boston: Artech House, 2015.

[54] S. Mikki and Y. Antar, "The antenna current Green's function as an alternative method to conventional full-wave analysis solvers: An outline," in 2015 IEEE MTT-S International Conference on Numerical Electromagnetic and Multiphysics Modeling and Optimization (NEMO), Aug 2015, pp. 1-3.

[55] W. C. Gibson, The Method of Moments in Electromagnetics. CRC Press, 2015.

[56] A. Ramm, "Theoretical and Practical Aspects of Singularity and Eigenmode Expansion Methods," IEEE Transactions on Antennas and Propagation, vol. 28, no. 6, pp. 897-901, Nov 1980.

[57] F. Sarrazin, S. Mikki, Y. Antar, P. Pouliguen, and A. Sharaiha, "Study of Dipole Antennas' Characteristic Modes Through the Antenna Current Green's Function and the Singularity Expansion Method," in 2015 9th European Conference on Antennas and Propagation 
(EuCAP), May 2015, pp. 1-2.

[58] A. M. Alzahed, S. Mikki, Y. M. Antar, M. Clénet, and S. Jovic, "Characterization of a rectangular patch antenna using ACGF-SEM approach," in 2016 IEEE Conference on Antenna Measurements \& Applications (CAMA). IEEE, 2016, pp. 1-3.

[59] — - "The ACGF-SEM approach to electromagnetic radiation with applications in radar and inverse modeling," in Proc. Int. Union Radio Sci. General Assem. Sci. Symp. (URSI), 2017.

[60] A. Alzahed, Analysis of Electromagnetic Systems Using the Antenna Current Green's function $(A C G F)$ and Machine Learning. Royal Military College of Canada: Ph.D Dissertation, 2019.

[61] A. M. Alzahed, S. M. Mikki, and Y. M. Antar, "Electromagnetic deep learning technology for radar target identification," in 2019 IEEE International Symposium on Antennas and Propagation and USNC-URSI Radio Science Meeting. IEEE, 2019, pp. 579-580.

[62] A. Alzahed, S. Mikki, and Y. Antar, "Electromagnetic machine learning for inverse modeling using the spatial singularity expansion method," IEEE Journal on Multiscale and Multiphysics Computational Techniques, pp. 1-1, 2020.

[63] S. Mikki and A. Kishk, "Theory and Applications of Infinitesimal Dipole Models for Computational Electromagnetics," IEEE Transactions on Antennas and Propagation, vol. 55, no. 5, pp. 1325-1337, May 2007.

[64] S. Mikki, S. Clauzier, and Y. Antar, "A correlation theory of antenna directivity with applications to superdirective arrays," IEEE Antennas and Wireless Propagation Letters, vol. 18 , no. 5, pp. 811-815, May 2019 